\title{
AN ALGEBRAIC MODEL OF TRANSITIVE DIFFERENTIAL GEOMETRY
}

\author{
VICTOR W. GUILLEMIN AND SHLOMO STERNBERG ${ }^{1}$
}

One of the basic assumptions that is frequently made in the study of geometry (or physics) is that of homogeneity. It is assumed that any point can be moved into any other by a transformation preserving the underlying geometrical structure (e.g., by a Euclidean motion in Euclidean geometry, an inhomogeneous Lorentz transformation in special relativity, etc.). The problem we shall treat in this paper is that of describing the possible differential geometries which are homogeneous or transitive in the above sense. We prefer the word "transitive" as "homogeneous" already has a technical meaning. It should be noted at the outset that we will only discuss the local problem, regarding two geometries as the same if they are locally the same. The problem of describing the global possibilities for a fixed geometrical structure is a problem of an entirely different order and is usually extremely interesting and difficult (the problem of moduli for Riemann surfaces, the Clifford-Klein Raum problem, etc.). Actually, we won't even handle the local problem which involves various technical difficulties such as solving partial differential equations, worrying about domains of definition, etc. What we shall do is construct an algebraic model which will illustrate all the crucial geometric notions.

Before giving a precise statement to the problem let us examine the situation in two dimensions. In order to list all the possible transitive geometries we must first list the various types of differential geometry (projective, conformal, Riemannian, etc.) and then list the possibilities for each type. For instance, the Korn-Lichtenstein theorem (asserting the existence of isothermal coordinates (cf. [10] or $[25]))$ implies that all conformal differential geometries in two dimensions are locally equivalent. In other words there is only one conformal differential geometry and it is transitive.

How many different transitive Riemannian geometries are there in two dimensions? Here we are faced with a choice. The professional differential geometer will answer that there is a continuum, each parametrized by a real number-the curvature. Indeed a sphere of

An address delivered by Shlomo Sternberg before the Boulder meeting of the Society on August 29, 1963, by invitation of the Committee to Select Hour Speakers for Summer and Annual Meetings; received by the editors August 25, 1963.

1 The research of the first author was supported in part by a grant from the National Science Foundation, and the research of the second author was supported in part by the Sloan Foundation and by NSF Grant GP-127. 
radius one is clearly not isometric to a sphere of radius one thousand. On the other hand, the classical geometer will say that all spherical geometries are the "same." What is at issue is the following: let $\phi_{r}$ be the dilatation map of the sphere, $S_{1}$, of radius one onto the sphere, $S_{r}$, of radius $r$. If $r \neq 1$ then $\phi_{r}$ is not an isometry. On the other hand $\phi_{r}$ does carry the group of motions of one sphere isomorphically onto the group of motions of the second. That is, if $T_{1}$ is any isometry of $S_{1}$ then $\phi_{r} \circ T_{1}=T_{r} \circ \phi_{r}$ where $T_{r}$ is an isometry of $S_{r}$. If we regard two geometries as the same if they are the same in this sense then there are essentially only three possibilities for transitive two-dimensional Riemannian geometries: their groups of automorphisms are $O(3)$ (spherical geometry), the Euclidean group (Euclidean geometry) or $S l(2)$ (hyperbolic geometry).

We can adopt a still more algebraic attitude and regard $O(3)$ acting on the sphere and $O(3)$ acting on itself via the adjoint representation as two different representations of the same abstract object, $O(3)$. We will therefore be concerned with classifying transitive geometries relative to three increasingly loose notions of equivalence which we may call "geometric equivalence," "algebraic equivalence," and "isomorphism." The precise definitions will be given later on.

By now the reader must suspect that there is a close connection between the problems we shall treat and the (finite and infinite) groups of Lie and Cartan. Most of the results we shall present are contained, implicitly and explicitly in the fundamental papers [3], [4] and [5] of E. Cartan. In fact, we shall present an algebraic model for the transitive groups and this paper might well serve as an introduction to this subject. For a translation into modern language of many of the ideas of Cartan on this subject see [15], [27], or [29, Chapter VII]. In these papers, some of the algebraic notions that are introduced here in a formal way are given geometrical definitions and interpretations.

Our algebraic model is constructed as follows: we study the geometry by studying its "infinitesimal automorphisms." We replace each infinitesimal automorphism (which is a vector field) by its Taylor expansion about a point. We are thus led to the study of formal (or, better, formal power series) vector fields.

1. In all the algebraic considerations which follow we shall not specify the ground field over which we operate. In the applications we have in mind it will always be the real or complex numbers. Our arguments will be valid for any field of characteristic zero. As we will make free use of symmetrization and anti-symmetrization operators 
(which involve dividing by $k !$ ), there may be some technical difficulties in extending our results to the case of finite characteristic.

Let $V$ be a finite dimensional vector space. We denote by $F\left\{V^{*}\right\}$ the ring of formal power series over $V$. It is a local ring whose maximal ideal, $F^{0}\left\{V^{*}\right\}$, consists of those formal power series which vanish at the origin (i.e., which have no constant term). We denote by $F_{k}\left\{V^{*}\right\}$ the ideal consisting of those power series vanishing to order $k$ and remark that the sets $F_{k}\left\{V^{*}\right\}$ form a sequence of neighborhoods of the origin in the natural topology on $F\left\{V^{*}\right\}$.

We denote by $D(V)$ the Lie algebra of all continuous derivations of $F\left\{V^{*}\right\}$. Let $\partial / \partial x^{1}, \cdots, \partial / \partial x^{n}$ be a basis of $V$ and $x^{1}, \cdots, x^{n}$ the dual basis of $V^{*}$. Since the polynomials are dense in $F\left\{V^{*}\right\}$, to specify an element $X$ of $D(V)$ it suffices to give the values $X x^{i}$ $=X^{i}\left(x^{1}, \cdots, x^{n}\right) \in F\left\{V^{*}\right\}$. We can thus write

$$
X=\sum_{i=1}^{n} X^{i}\left(x^{1}, \cdots, x^{n}\right) \frac{\partial}{\partial x^{i}} .
$$

It is therefore clear that an element of $D(V)$ can be regarded as a "formal vector field." If $X=\sum X^{i}\left(\partial / \partial x^{i}\right)$ and $Y=\sum Y^{i}\left(\partial / \partial x^{i}\right)$ are two elements of $D(V)$ then $[X, Y]=Z=\sum Z^{i}\left(\partial / \partial x^{i}\right)$ where

$$
Z^{i}=\sum \frac{\partial X^{i}}{\partial x^{j}} Y^{j}-\frac{\partial Y^{i}}{\partial x^{j}} X^{j}
$$

$D(V)$ will be our analogue of the "algebra" of all infinitesimal automorphisms of a manifold $M$. We wish to study subalgebras of $D(V)$ corresponding to the infinitesimal automorphism of some transitive geometrical structure on $M$. To see which subalgebras of $D(V)$ we need, we study the structure of $D(V)$ a little more closely.

Notice that $D(V)$ has a natural filtration: define the spaces $D^{k}(V) \subset D(V)$ by

$$
X \in D^{k}(V) \quad \text { if } X f \in F^{k}\left\{V^{*}\right\} \quad \text { for all } f \in F\left\{V^{*}\right\} .
$$

In terms of the representation (1.1) an element $X$ belongs to $D^{k}(V)$ if and only if all its $X^{i}$ belong to $F^{k}\left\{V^{*}\right\}$. If we set $D^{-1}(V)=D(V)$ then

$$
\left[D^{k}(V), D^{l}(V)\right] \subset D^{k+l}(V)
$$

as can be easily verified from the definitions. In particular, $D^{\circ}(V)$ is a subalgebra of $D(V)$ and $D^{k}(V)$ is an ideal in $D^{0}(V)$.

The space $D^{k}(V) / D^{k+1}(V)$ can be naturally identified with $V \otimes S^{k+1}\left(V^{*}\right)$ and (1.3) shows that the bracket operation on $D(V)$ induces a pairing (which we continue to denote by bracket) of 
$V \otimes S^{k+1}\left(V^{*}\right) \times V \otimes S^{l+1}\left(V^{*}\right) \rightarrow V \otimes S^{k+l+1}\left(V^{*}\right):$

$$
\left[V \otimes S^{k+1}\left(V^{*}\right), V \otimes S^{l+1}\left(V^{*}\right)\right] \subset V \otimes S^{k+l+1}\left(V^{*}\right) \text {. }
$$

We can, in fact, regard $V \otimes S^{k+1}\left(V^{*}\right)$ as a subspace of $D(V)$ (consisting of those $X=\sum X^{i}\left(\partial / \partial x^{i}\right)$ where the $X^{i}$ are homogeneous polynomials of degree $k+1$ ). Then the pairing of (1.4) is just the restriction of the bracket operation of $D(V)$ and the subalgebra

$$
P(V)=V+V \otimes V^{*}+V \otimes S^{2}\left(V^{*}\right)+\cdots
$$

(direct sum)

is just the algebra of polynomial vector fields.

A case of (1.4) of particular importance for us is where $k=-1$. We thus get a pairing

$$
\left[V, V \otimes S^{l+1}\left(V^{*}\right)\right] \subset V \otimes S^{l}\left(V^{*}\right)
$$

and the previous discussion shows that this pairing is the obvious one:

$$
[v, w \otimes f]=w \otimes f_{v}
$$

where $v, w \in V$ and $f$ is a homogeneous polynomial and where $f_{v}$ is the derivative of $f$ with respect to $v$.

For any subalgebra, $L$, of $D(V)$, we set

$$
\begin{aligned}
& L^{i}=L \cap D^{i}(V), \\
& g_{L}^{i}=L^{i} / L^{i+1}
\end{aligned}
$$

so that $g_{L}^{i}$ can be regarded as a subspace of $V \otimes S^{i+1}\left(V^{*}\right)$. Since $L$ is a subalgebra we have

$$
\left[g_{L}^{k}, g_{L}^{l}\right] \subset g_{L}^{k+l}
$$

In particular, $g_{L}^{0} \subset V \otimes V^{*}$ is a subalgebra of $V \otimes V^{*}$ which we shall call the linear isotropy algebra of $L$ and denote simply by $g$.

The space $g_{L}^{-1}$ is a subspace of $V$. We shall say that $L$ is transitive if it is all of $V$. Thus $L$ is transitive if $L / L_{0}=V$. (Geometrically this means that we can "move infinitesimally" in every direction by an element of $L$.)

The filtration $\left\{D^{k}(V)\right\}$ provides a system of neighborhoods for a topology on $D(V)$ and (1.3) shows that the bracket operation is continuous relative to this topology. Our first objects of study will be the transitive subalgebras of $D(V)$ which are closed in the above topology.

Let $\phi$ be a formal power series isomorphism of $V$ onto $W$. (In terms of bases of $V$ and $W$ a formal power series isomorphism is given by $n$ formal power series $y^{1}=\phi^{i}\left(x^{1}, \cdots, x^{n}\right)$ where the $\phi^{i}$ have zero con- 
stant term and $\operatorname{det}\left|\partial \phi^{i} / \partial x^{j}\right|_{0, \ldots, 0} \neq 0$. More abstractly, a formal power series isomorphism $\phi$ is given by an isomorphism, $\phi^{*}$ of $F\left\{W^{*}\right\}$ onto $F\left\{V^{*}\right\}$ carrying $F^{0}\left\{W^{*}\right\}$ into $F^{0}\left\{V^{*}\right\}$.) The map $\phi$ induces (or is defined by) an isomorphism $\phi^{*}$ of $F\left\{W^{*}\right\}$ onto $F\left\{V^{*}\right\}$ and therefore an isomorphism, $\phi_{*}$, of $D(V)$ onto $D(W)$. For any $X \in D(V)$ and $f \in F\left\{W^{*}\right\}$ we have

$$
\left(\phi_{*} X\right) f=\phi^{-1 *}\left(X \phi^{*} f\right) \text {. }
$$

Let $L$ be a subalgebra of $D(V)$ and $M$ a subalgebra of $D(W)$. Let $\phi$ be a formal power series isomorphism of $V$ onto $W$. We say that $\phi$ is an equivalence of $L$ with $M$ if $\phi_{*}(L)=M$. One of the main problems we shall be concerned with is that of deciding when two closed transitive algebras are equivalent. That is, we wish to obtain a complete set of invariants of closed transitive algebras under equivalence. This corresponds to the classification of the transitive geometries under "algebraic equivalence" mentioned in the introduction.

2. As we observed in the introduction, the classification of transitive geometries splits into two steps: first the list of the different types of geometry then the classification of the various geometries of each type. We expect this to be reflected in our model. Accordingly, in this section we define the "type" of geometry associated with each transitive closed formal power series algebra and then discuss the various possible types.

Our approach will be based on the following remark: let $\phi$ be a formal power series isomorphism of $V$ onto $W$ and let $l_{\phi}$ be the associated linear map of $V$ onto $W$. (Thus $l_{\phi}$ is the "Jacobian of $\phi$ at the origin.") Since $l_{\phi}$ is a linear isomorphism, it induces a linear map of $V \otimes S^{k+1}\left(V^{*}\right)$ onto $W \otimes S^{k+1}\left(W^{*}\right)$ which we shall also denote by $l_{\phi}$ (by abuse of language). Let $L$ be a subalgebra of $D(V)$ and $M$ be a subalgebra of $D(W)$ and suppose that $\phi_{*}(L)=M$. Now $g_{L}^{k}$ $C V \otimes S^{k+1}\left(V^{*}\right)$ and $g_{M}^{k} \subset W \otimes S^{k+1}\left(W^{*}\right)$. We claim that

$$
\stackrel{k}{g_{M}}=l_{\phi}\left(g_{L}^{k}\right) \text {. }
$$

To see this it suffices to observe that an element of $D^{k}(W) / D^{k+1}(W)$ is determined by the map it induces of $F^{0}\{W\} / F^{1}\{W\}$ into $F^{k}\{W\} / F^{k+1}\{W\}$. For any $f \in F^{0}\{W\}$ we have $\phi^{*} f=f \circ l_{\phi} \bmod F^{1}$ so that, by (1.8)

$$
\left(\phi_{*} X\right) f=l_{\phi}^{-1}\left(X\left(f \circ l_{\phi}\right)\right) \bmod F^{k+1}\left\{W^{*}\right\} .
$$

Equation (2.1) shows that (up to linear isomorphism of the space $V)$ the spaces $g_{L}^{k} \subset V \otimes S^{k+1}\left(V^{*}\right)$ are invariants of $L$. The sequence 
of spaces $\left\{g_{L}^{k}\right\}$ (actually, as we shall see, only a finite number of them) describes the type of geometry given by $L$.

Given a sequence of spaces $g^{i} \subset V \otimes S^{i+1}\left(V^{*}\right)$ (with $g^{-1}=V$ ) what conditions must they satisfy in order that there exist an $L$ with $g^{i}=g_{L}^{i}$ ? Obviously, condition (1.7) must be satisfied. That is, under the pairing (1.4) we must have $\left[g^{k}, g^{l}\right] \subset g^{k+l}$. Conversely, if the $g^{i}$ satisfy (1.7) then the space $V+g^{0}+g^{1}+\cdots+g^{k}+\cdots$ is a subalgebra of $P(V)$. Its closure is thus a transitive closed algebra $L$ with $g^{i}=g_{L}^{i}$. We now examine condition (1.7) a little more closely, especially the case $k=-1$. For this purpose we introduce the following definitions:

Definition 2.1. Let $P$ and $Q$ be vector spaces and $h$ a subspace of $\operatorname{Hom}(P, Q)$. The space $h^{(1)} \subset \operatorname{Hom}(P, h)$, called the first prolongation of $h$, consists of all $T \in \operatorname{Hom}(P, h)$ satisfying

$$
T(u) v=T(v) u \quad \text { for all } u, v \in P .
$$

Here $T(u) \in h=\operatorname{Hom}(P, Q)$ so $(2.2)$ makes sense. When $P$ is finite-dimensional (the only case we shall consider) we can identify $\operatorname{Hom}(P, Q)$ with $Q \otimes P^{*}$ and rewrite $(2.2)$ as

$$
h^{(1)}=\operatorname{Hom}(P, h) \cap Q \otimes S^{2}\left(V^{*}\right) .
$$

Definition 2.2. We define $h^{(k)}$ inductively by setting $h^{(k+1)}=h^{(k)}{ }^{(1)}$. The space $h^{(k)}$ is called the kth prolongation of $h$. We say that $h$ is of finite type if $h^{(k)}=0$ for some (and hence all larger) $k$. Otherwise we say that $h$ is of infinite type.

Recalling that for transitive $L$ we have $g_{L}^{-1}=V$ and using (1.5) we see that condition (1.7) for the case $k=-1, l=i+1$ can be expressed as

$$
g^{i+1} \subset g^{i^{(1)}} .
$$

Lemma 2.1. Let $P$ and $Q$ be finite-dimensional vector spaces and let $h^{0} \mathrm{CHom}(P, Q)$ and $h^{i}(i \geqq 1)$ be a sequence of spaces satisfying

$$
h^{i+1} \subset h^{i^{(1)}} \text {. }
$$

Then there exists an integer $k$ such that

$$
h^{k+s}=h^{(s)} \quad \text { for all } s \geqq 1 .
$$

This lemma is an immediate consequence of the Hilbert basis theorem, cf. [27] for the proof which we shall not repeat here.

In view of (2.3) and the lemma, we can associate to each transitive algebra $L$ the smallest integer, $r$, such that $g_{L}^{r+s}=g_{L}^{r(s)}$ for all $s \geqq 1$. The integer $r+1$ is called the geometric order of $L$. Our first set of invariants of $L$ thus consists of an integer $r$, together with a finite se- 
quence $g_{L}^{0}, \cdots, g_{L}^{r}$ satisfying (1.7). This describes the "type of geometry" whose infinitesimal automorphisms are the elements of $L$.

Conversely, suppose we are given a finite sequence $g^{1}=V, g^{0}, \cdots, g^{r}$ where $g^{i} \subset V \otimes S^{i+1}\left(V^{*}\right)$ such that (1.7) holds whenever $k, l$ and $k+l$ are all less than $r$. We assert that there is an $L$ of order $r$ with $g_{L}^{i}=g^{i}$ $(i \leqq r)$. In fact, just set $g^{r+s}=g^{r^{(s)}}$ and check that now the $g^{i}$ satisfy (1.7) for all values of $i$. This follows by repeated application of Jacobi's identity and those cases of (1.7) which we do know. For instance, suppose $k+l=r+1$ and we wish to verify $\left[g^{k}, g^{l}\right] \subset g^{r+1}=g^{r^{(1)}}$. By definition, this amounts to showing that $\left[V,\left[g^{k}, g^{l}\right]\right] \subset g^{r}$. But by Jacobi's identity

$$
\begin{aligned}
{\left[V,\left[g^{k}, g^{l}\right]\right] } & =\left[\left[V, g^{k}\right], g^{l}\right]+\left[g^{k},\left[V, g^{l}\right]\right] \\
& \subset\left[g^{k-1}, g^{l}\right]+\left[g^{k}, g^{l-1}\right] \\
& \subset g^{r}
\end{aligned}
$$

by (1.7). The general argument proceeds similarly and will be left to the reader.

Thus starting with $V, g^{0}, \cdots, g^{r}$ satisfying (1.7) for $k+l \leqq r$ we obtain a sequence $\left\{g^{i}\right\}$ satisfying (1.7) for all $i$ and thus an $L$ with $g_{L}^{i}=g^{i}$.

DEFinition 2.3. The transitive closed formal algebra obtained in the above way from $g^{0}, \cdots, g^{r}$ is called the flat algebra of type $g^{0}, \cdots, g^{r}$ and will be denoted by $L_{g^{0}}, \ldots, g^{r}$.

Of course, instead of taking $g^{r+t}=g^{(t)}$, we might have considered the closure, $L^{\prime}$, of the algebra generated by $V, g^{0}, \ldots, g^{r}$, in $P(V)$. Arguments similar to the above show that $g_{L^{\prime}}^{i}=g^{2}$ for $i \leqq r$. However $L^{\prime}$ is smaller than $L$ and will not be of order $r$ in general. Frequently there isn't much room between $L^{\prime}$ and $L_{g}{ }^{0}, \ldots, g^{r}$, and one can list the possible types geometry which "start" with $g^{0}, \cdots, g^{r}$. For instance there are only four possibilities for geometries whose $g^{0}$ is all of $V \otimes V^{*}$ (see below).

A distinction, already made by Lie, is very important in geometrical applications:

Definition 2.4. A subalgebra $L \subset D(V)$ is called finite if $\operatorname{dim} L<\infty$ and infinite otherwise.

It is clear that $L$ is infinite if and only if $L^{k} \neq 0$ for all $k$. For transitive $L$ this can only happen if $g_{L}^{k} \neq 0$ for all $k$. By Lemma 2.1 and Definition 2.2 we can therefore assert

Proposition 2.1. A transitive closed subalgebra, $L \subset D(V)$, of geometric order $r$ is finite or infinite according as $g_{L}^{r}$ is of finite or infinite type. 
3. Before proceeding with the general theory, we pause to give some examples illustrating the notions of the previous two sections.

(A) Riemannian (or Lorentzian) geometries. Let ( , ) be a nondegenerate symmetric bilinear form on $V$ (of arbitrary signature). Let $o(V)$ be the orthogonal algebra of $($,$) . That is, A \in o(V)$ if and only if

$$
(A u, v)+(u, A v)=0
$$

for all $u, v \in V$. We assert that $o(V)^{(1)}=0$. In fact, for any $T \in o(V)^{(1)}$, and any $u, v, w \in V$ we have, by $(3.1)$, the symmetry of $($,$) and$ (2.2),

$$
\begin{aligned}
(T(w) v, u) & =(T(v) w, u)=-(T(v) u, w) \\
& =-(T(u) v, w)=(T(u) w, v) \\
& =(T(w) u, v)=-(T(w) v, u) .
\end{aligned}
$$

Thus $(T(w) u, v)=0$ which implies that $T=0$ as $($,$) is nonsingular.$

Thus, in particular, all Riemannian geometries are of finite type. This is the algebraic analogue of the famous theorem of Myers and Steenrod asserting that the group of automorphisms of a Riemann manifold is a (finite dimensional) Lie group. Actually this geometrical fact is a fairly easy consequence of the algebraic one via an elegant argument due to Kobayashi. For a presentation of these results cf. [15] or [29, Chapter VII, \$4].

The flat algebra $L_{o(V)}$ is, of course, nothing other than the algebra of infinitesimal Euclidean motions.

(B) Conformal geometries. Let $($,$) be as before and let c o(V)$ denote its conformal algebra. That is, $A \in c o(V)$ if and only if

$$
(A u, v)+(u, A v)=\lambda(u, v) \quad \text { for all } u, v \in V
$$

where $\lambda$ is some scalar depending on $A$. (Notice that for those $u, v$ with $(u, v)=0(3,2)$ reduces to (3.1).) We now compute $c o(V)^{(1)}$. For any $T \in \operatorname{co}(V)^{(1)}$ we get a linear form $\lambda(\cdot)$ defined by

$$
(T(w) u, v)+(u, T(w) v)=\lambda(w)(u, v) .
$$

We thus have a linear map of $c o(V)^{(1)} \rightarrow V^{*}$. This map is clearly injective since a $T$ lying in its kernel would lie in $o(V)^{(1)}$ and thus vanish by (A). Let us show that it is also surjective. To this effect we observe that $($,$) induces an isomorphism of V$ onto $V^{*}$. Thus $u \in V$ is mapped onto $u^{*} \in V^{*}$ where $\left\langle v, u^{*}\right\rangle=(v, u)$. If we replace $($,$) by \lambda($,$) then under the new isomorphism u$ gets sent into $\lambda u^{*}$. In particular, the isomorphism of $V \otimes V^{*}$ onto $V^{*} \otimes V$ induced by 
$($,$) is independent of the scalar \lambda$, i.e., is an invariant of $c o(V)$. Let us denote this isomorphism by $\phi$. For any $u^{*} \in V^{*}$ let $\gamma\left(u^{*}\right)$ $\in \operatorname{Hom}(V, \operatorname{Hom}(V, V))$ be defined by

$$
\gamma\left(u^{*}\right)(v)=v \otimes u^{*}-\phi\left(u^{*} \otimes v\right)+\left\langle v, u^{*}\right\rangle I
$$

where $I$ is the identity. We claim that $\gamma\left(u^{*}\right) \in c o(V)^{(1)}$. In fact,

$$
\gamma\left(u^{*}\right)\left(v_{1}\right) v_{2}=\left(v_{1}, u\right) v_{2}+\left(v_{2}, u\right) v_{1}-\left(v_{1}, v_{2}\right) u \text {. }
$$

Thus $c o(V)^{(1)}$ is isomorphic to $V^{*}$.

Let us compute $c o(V)^{(2)}$. For any four vectors $u, v, x$, and $y$, and for any $T \in \operatorname{co}(V)^{(2)}$ we have

$$
(T(u, v) x, y)+(x, T(u, v) y)=\lambda_{u v}(x, y) .
$$

Here $\lambda_{u v}$ is a symmetric bilinear form in $u$ and $v$ depending on $T$. If $\lambda$ vanishes then $T$ belongs to $o(V)^{(2)}$ and hence must vanish. Since $\lambda$ is symmetric, to show that a given $\lambda$ vanishes it suffices to show that $\lambda_{u u}$ vanishes identically. Let us choose $u$ and $v$ with $(u, v)=0$. Then, since (3.2) reduces to (3.1) in this case, we have

$$
\begin{aligned}
\lambda_{u u}(v, v) & =2(T(u u) v, v) \\
& =2(T(u v) u, v) \\
& =-2(T(u v) v, u) \\
& =-2(T(v v) u, u) \\
& =-\lambda_{v v}(u, u) .
\end{aligned}
$$

Thus for every pair of orthogonal vectors we have $\lambda_{u u}(v, v)$ $=-\lambda_{v v}(u, u)$. If $\operatorname{dim}(V) \geqq 3$ this obviously implies that $\lambda=0$. In other words $c o(V)^{(2)}=0$ if $\operatorname{dim} V \geqq 3$.

If $\operatorname{dim} V=2$ then it is easy to check that $c o(V)$ is of infinite type. This accounts for the difference in conformal geometry between two and more dimensions.

If $\operatorname{dim} V>2$ then the flat algebra $L_{c o(V)} \sim V+c o(V)+V^{*}$ is known as the Möbius algebra. It can be identified with the Lie algebra of the group of conformal transformations of the sphere whose dimension is $\operatorname{dim}(V)$. Notice that there are two types of geometries with $g^{0}=c o(V)$. The conformal geometries whose geometrical order is one (thus with $g^{1}=c o(V)^{(1)}$ ) and the geometries of order 2 where $g^{1}=0$. There are no further possibilities since $c o(V)^{(1)} \sim V^{*}$ is irreducible under the action of $c o(V)$ and the case $k=0$ of (1.7) implies that $g_{L}^{l}$ must be invariant under the action of $g_{L}^{0}$. The flat algebra $L_{c e(V), 0}$ is just the Lie algebra of the group of similarity transformations of Euclidean geometry. 
(C) Let us consider the various types of geometries whose $g^{0}=g l(V)$ $=V \otimes V^{*}$. There is obviously only one first order structure-the full algebra $D(V)$. To study the possible second order structures we must describe the decomposition of $V \otimes S^{2}\left(V^{*}\right)$ into subspaces invariant under the action of $g l(V)$. One obvious such subspace is $s l(V)^{(1)}$. The flat algebra $L_{g l(V) s l(V)^{(1)}}$ consists of all formal vector fields with constant divergence. The corresponding geometrical structure is given by a volume element determined up to constant factor. It turns out, using the methods to be developed below, that this is the only $g l(V), s l(V)^{(1)}$ structure (and indeed the only structure starting with $g l(V), \operatorname{sl}(V)^{(1)}$ (cf. [27, Chapter V])).

Since $g l(V)$ is reductive, we can find an invariant complement to $\operatorname{sl}(V)^{(1)}$ in $V \otimes S^{2}\left(V^{*}\right)$. We now describe it explicitly. Let $\sigma$ be the map of $V \otimes V^{*} \otimes V^{*}$ onto $V \otimes S^{2}\left(V^{*}\right)$ given by $\sigma\left(v \otimes u^{*} \otimes w^{*}\right)$ $=v \otimes\left(u^{*} \otimes w^{*}+w^{*} \otimes u^{*}\right)$. For any $u^{*} \in V^{*}$, let $\gamma\left(u^{*}\right)=\sigma\left(I \otimes u^{*}\right)$, where $I \in V \otimes V^{*}$ is the identity transformation. Then

$$
\gamma\left(u^{*}\right)(v)=v \otimes u^{*}+\left\langle v, u^{*}\right\rangle I .
$$

The map $\gamma$ is clearly injective and the space $\gamma\left(V^{*}\right)$ is an invariant subspace of $g l(V)^{(1)}$ which we shall denote by $p^{1}$. It is obviously

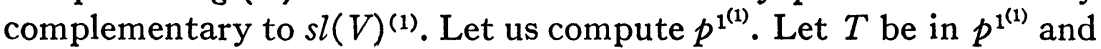
$v_{1}, v_{2} \in V$. Let $T_{v_{1}}=\gamma\left(u_{1}^{*}\right)$ and $T_{v_{2}}=\gamma\left(u_{2}^{*}\right)$. Then

$$
T_{v_{1} v_{2}}=v_{2} \otimes u_{1}^{*}+\left\langle v_{2}, u_{1}^{*}\right\rangle I
$$

and

so that

$$
T_{v_{2} v_{1}}=v_{1} \otimes u_{2}^{*}+\left\langle v_{2}, u_{2}\right\rangle I
$$

$$
T_{v_{1} v_{2} v_{2}}=2\left\langle v_{2}, u_{1}^{*}\right\rangle v_{2}=T_{v_{2} v_{1} v_{2}}=2\left\langle v_{2}, u_{2}^{*}\right\rangle v_{1}+\left\langle v_{1}, u_{2}^{*}\right\rangle v_{2} .
$$

If $\operatorname{dim} V>1$ then for any nonzero $v_{1}$ we can choose $v_{2}$ to be linearly independent of $v_{1}$. Then the last equation implies that $\left\langle v_{2}, u_{2}^{*}\right\rangle=0$ and $\left\langle v_{1}, u_{2}^{*}\right\rangle=2\left\langle v_{2}, u_{1}^{*}\right\rangle$. Interchanging $v_{1}$ and $v_{2}$ we conclude that $u_{1}^{*}=0$. Thus $p^{1^{(1)}}=0$. For $\operatorname{dim} V>1$ the algebra

$$
L_{o l(V), p^{1}}=V+g l(V)+p^{1} \quad\left(=V+g l(V)+V^{*}\right)
$$

is the algebra of infinitesimal collinations of projective space.

It turns out, cf. [27, Chapter V], that there are four possible geometries with $g^{0}=g l(V)$, namely $D(V), L_{o l(V), s l(V)^{1}}$, which are infinite, projective geometry and affine geometry $\left(=L_{g l(V), 0}\right)$ which are finite.

4. We return to the general theory. The next main problem on the agenda is how to decide when two $L$ 's with the same $g^{i}$ are equivalent. 
In order to develop the appropriate machinery we first consider a special case of this problem, namely, when is a given $L$ flat? That is, is $L$ equivalent to $L_{g_{L}}{ }^{0} \cdots, o_{L}{ }^{r}$ ? If $L$ is flat then it has the same algebraic structure as the flat algebra. In particular, it must be possible to choose a complementary abelian subalgebra, $\bar{L}$, to the subalgebra $L^{0}$. Let us examine the obstructions to the choice of such an $\bar{L}$.

Let $\tilde{V}$ be some (vector space) complement to $L^{0}$ in $L$. For any $u, v$ etc. in $V$ we denote by $\tilde{u}, \tilde{v}$, etc. the element of $\tilde{V}$ with $\tilde{u} / L^{0}=u$, $\tilde{v} / L^{0}=v$ and so on. Our choice of $\tilde{V}$ induces a map $\tilde{c}$ of $V \wedge V \rightarrow V$ defined by setting

$$
\tilde{c}(u \wedge v)=w \text { where }[\tilde{u}, \tilde{v}]=\tilde{w}+l \text { with } l \in L^{0} .
$$

Let us see how much this map depends on the choice of $\tilde{V}$. Let $\hat{V}$ be a second such choice. The pair $\tilde{V}, \hat{V}$ yields a map $S\left(=S_{\tilde{V}}, \hat{V}\right)$ of $V \rightarrow g$ defined as follows: for each $v \in V$ we have $\tilde{v}-\hat{v} \in L^{0}$. The image of $\tilde{v}-\hat{v}$ in $L^{0} / L^{1}=g$ is what we call $S(v)$. Now let us compare $\tilde{z}$ and $\hat{t}$. For any $u$ and $v \in V$ we have

$$
\begin{aligned}
{[\tilde{u}, \tilde{v}]-[\hat{u}, \hat{v}] } & =[\tilde{u}-\hat{u}, \tilde{v}]+[\hat{u}, \tilde{v}-\hat{v}] \\
& =\widehat{S(u) \cdot v}-\widehat{S(v) u} \bmod L^{0} .
\end{aligned}
$$

We thus have

$$
\tilde{c}(u \wedge v)-\hat{c}(u \wedge v)=S(u) \cdot v-S(v) \cdot u .
$$

Conversely, given any $S \in \operatorname{Hom}(V, g)$ it is clear that we can choose $\tilde{V}$ and $\hat{V}$ so that $S=S_{\tilde{V}} . \hat{v}$ so that (4.2) represents the actual degree of indeterminancy of $\tilde{c}$. It is sometimes more convenient to write $(2.1)$ in more succinct form: let $\partial$ be the map of $g \otimes V^{*}=\operatorname{Hon}(V, g)$ $\rightarrow V \otimes \wedge^{2}\left(V^{*}\right)=\operatorname{Hom}(V \wedge V, V)$ given by $\partial(S)(u \wedge v)=S(u) v-S(v) u$. Then we have

$$
\tilde{c}-\hat{c}=\partial\left(S_{\tilde{V}}, \hat{V}\right) .
$$

In particular, $\tilde{c}-\hat{c} \in \partial\left(g \otimes V^{*}\right)$. This means that we get a well-defined element of $V \otimes \Lambda^{2}\left(V^{*}\right) / \partial\left(g \otimes V^{*}\right)$ which we call the first order structure constant of $L$. It is clear that if $L$ is flat, this constant must vanish.

The map $\tilde{z}$ is not completely arbitrary. We have the Jacobi identity of $L$,

$$
[[\tilde{u}, \tilde{v}], \tilde{w}]+[[\tilde{v}, \tilde{w}], \tilde{u}]+[[\tilde{w}, \tilde{u}], \tilde{v}]=0,
$$

to take into account. Let us define the map $\tilde{b} \in \operatorname{Hom}(V \wedge V, g)$ $=g \otimes \Lambda^{2}\left(V^{*}\right)$ by letting $\tilde{b}(u \wedge v)$ be the image in $L^{0} / L^{1}$ of the element

$$
\lfloor\tilde{u}, \tilde{v}]-\overparen{c(u \wedge v)}
$$


of $L^{0}$. Then Jacobi's identity implies

$$
\begin{aligned}
\tilde{c}(\tilde{c}(u \wedge v) \wedge w) & +\tilde{c}(\tilde{c}(v \wedge w) \wedge u)+\tilde{c}(\tilde{c}(w \wedge u) \wedge v) \\
& +\tilde{b}(u \wedge v) w+\tilde{b}(v \wedge w) u+\tilde{b}(w \wedge u) v=0 .
\end{aligned}
$$

We shall call (4.4) Bianchi's identities. We can also write (4.4) in more succinct form : let $\tilde{c} \rightarrow \tilde{c}^{2}$ be the quadratic map of $\operatorname{Hom}(V \wedge V, V)$ $\rightarrow \operatorname{Hom}(V \wedge V \wedge V, V)$ given by

$\tilde{c}^{2}(u \wedge v \wedge w)=\tilde{c}(\tilde{c}(u \wedge v) \wedge w)+\tilde{c}(\tilde{c}(v \wedge w) \wedge u)+\tilde{c}(\tilde{c}(w \wedge u) \wedge v)$ and let $\partial: g \otimes \Lambda^{2}\left(Y^{*}\right) \rightarrow V \otimes \Lambda^{3}\left(V^{*}\right)$ be given by

$$
\partial(\tilde{b})(u \wedge v \wedge w)=b(u \wedge v) w+b(v \wedge w) u+b(w \wedge u) v
$$

Then we can rewrite (4.4) as

$$
\tilde{c}^{2} \in \partial\left(g \otimes \Lambda^{2}\left(V^{*}\right)\right) .
$$

It is an elementary algebraic exercise to verify that if $\tilde{c}$ satisfies (4.5) then so does $\hat{c}$ whenever $\tilde{c}$ and $\hat{c}$ are related by (4.3).

For any $X \in g$ choose an $\tilde{X} \in L^{0}$ whose image in $L^{0} / L^{1}=g$ is $X$. Applying Jacobi's identity to $[\tilde{X},[\tilde{u}, \tilde{v}]]$ shows that

$(4.6) X \tilde{c}(u \wedge v)=\tilde{c}(X u \wedge v)+\tilde{c}(u \wedge X v)+S(X)(u) v-S(X)(v) u$ where $S(X) \in \operatorname{Hom}(V, g)$. In terms of the induced action of $g$ on $V \otimes V^{*} \otimes V^{*}$ we can rewrite (4.6) as

$$
X \tilde{c}=\partial S(X) .
$$

Equations (4.2)-(4.6) should be called the (first order) Cartan structural equations of $L$. They are (modulo changes in notation) equations (19) and (20) of [3] or (19) and (20) of [7].

Suppose that $c=0$. We can thus choose a subspace $\tilde{V} \subset L$ so that $\tilde{c}=0$, i.e. $[\tilde{u}, \tilde{v}] \in L^{0}$ for all $u, v \in V$. For any such choice of $\tilde{V}$ we get a map $\tilde{c}^{1}$ of $V \wedge V \rightarrow g$ defined by

$$
\tilde{c}^{1}(u \wedge v)=[\tilde{u}, \tilde{v}] / L^{\prime} .
$$

Again, $\tilde{c}^{1}$ depends on our choice of $\tilde{V}$ and we can explicitly describe how much it depends on the choice. In order to give a convenient résumé of the appropriate facts we introduce some homological notation.

Consider the map of

$$
V \otimes \underbrace{V^{*} \otimes \cdots \otimes V^{*}}_{k \text {-times }} \otimes \underbrace{V^{*} \otimes \cdots \otimes V^{*}}_{l \text {-times }}
$$

into 


$$
V \otimes \underbrace{V^{*} \otimes \cdots \otimes V^{*}}_{(k-1) \text { times }} \otimes \underbrace{V^{*} \otimes \cdots \otimes V^{*}}_{(l+1) \text { times }}
$$

given by the associative law of tensor multiplication (move one factor past the big tensor product sign). Now $V \otimes S^{k}\left(V^{*}\right) \otimes \Lambda^{l}\left(V^{*}\right)$ can be regarded as a subspace of the first of these spaces and there is an obvious projection (given by symmetrization and anti-symmetrization) of the second of these spaces onto $V \otimes S^{k-1}\left(V^{*}\right) \otimes \Lambda^{l+1}\left(V^{*}\right)$. By restriction and projection we thus get a map, $\partial$, of $V \otimes S^{k}\left(V^{*}\right) \otimes \Lambda^{l}\left(V^{*}\right)$ into $V \otimes S^{k-1}\left(V^{*}\right) \otimes \Lambda^{l+1}\left(V^{*}\right)$. From the definitions, $\partial^{2}=0$. Now let $g^{0} \subset V \otimes V^{*}, g^{1}, \cdots, g^{i}, \cdots$ be a sequence of spaces satisfying (2.3). Since $g^{k} \subset V \otimes S^{k+1}\left(V^{*}\right)$ the space

$$
C^{k+1, l}=g^{k} \otimes \Lambda^{l}\left(V^{*}\right)
$$

is a subspace of $V \otimes S^{k+1}\left(V^{*}\right) \otimes \Lambda^{l}\left(V^{*}\right)$. We claim that $\partial C^{k+1, l}$ $\subset C^{k, l+1}$. In fact, for any $\xi \in C^{k+1, l}$ we have

$$
\partial \xi\left(v_{1}, \cdots, v_{l+1}\right)=\sum(-1) i \xi\left(v_{1}, \cdots \hat{v}_{i}, \cdots, v_{l}\right) v_{i}
$$

and each summand on the right lies in $g^{i-1}$ by virtue of (2.3). We thus get a differential operator $\partial$ mapping $C^{k+1, l} \rightarrow C^{k, l+1}$. The corresponding homology group $H^{k, l}\left(g^{0}, \cdots, g^{i}, \cdots\right)$ we shall call the Spencer homology groups of the geometrical type $\left(g^{0}, \cdots, g^{i}, \cdots\right)$. Although these groups are implicit in the work of Cartan they seem to have been first made explicit in the fundamental paper of Spencer [28] on deformation of structures. It turns out that the crucial obstructions to flatness lie in $H^{k, 2}$.

Lemma 4.1. Let $g^{0} \subset V \otimes V^{*}, g^{1}, \cdots, g^{i}, \cdots$ be a sequence of spaces satisfying (2.3). Then for sufficiently large $k, H^{k, l}\left(g^{0}, \cdots, g^{i}, \cdots\right)$ $=0$.

The proof of this lemma reduces to standard facts in commutative algebra (cf. [27] for the details). Notice that the case $l=1$ of the lemma is just Lemma 2.1.

An immediate consequence of Lemma 4.1 is that there are only a finite number of obstructions to the construction of an abelian complement to $L_{0}$. Actually we can say a lot more. Let $R=R(L)$ be the smallest integer such that $H^{k, 1}\left(g_{L}^{0}, \cdots, g_{L}^{i}, \cdots\right)=H^{k, 2}\left(g_{L}^{0}, \cdots\right.$, $\left.g_{L}^{i}, \cdots\right)=H^{k, 3}\left(g_{L}^{0}, \cdots, g_{L}^{i}, \cdots\right)=0$ for all $k \geqq R$. We call $R$ the structural order of $L$. It depends only on the geometrical type of $L$ and we clearly have $r \leqq R$. The theorem that we wish to assert says that a knowledge of $L$ "up to order $R$ " determines $L$ up to equivalence. To make this precise we introduce the following definition: 
Definition 4.1. Let $V$ be a finite dimensional vector space and $g \subset V \otimes V^{*}$ be a Lie algebra of linear transformations on $V$ and $c \in V \otimes \Lambda^{2}\left(V^{*}\right) / \partial\left(g \otimes V^{*}\right)$ where some (and hence every) $\tilde{c} \in V \otimes \wedge^{2}\left(V^{*}\right)$ with $\tilde{c} / \partial\left(g \otimes V^{*}\right)=c$ satisfies $(4.4)-(4.6)$. The triple $(V, g, c)$ is called a truncated Lie algebra. If $(W, h, d)$ is a second truncated Lie algebra, a bijective linear map, $l$, of $V \rightarrow W$ is called an isomorphism if $l(g)=h$ and $l(c)=d$ (where $l$ acts in the obvious way on the appropriate spaces).

Notice that if $L$ is a transitive subalgebra of $D(V)$ then there is an obvious $c^{k}$ such that $\left(L / L^{k}, g_{L}^{k}, c^{k}\right)$ is a truncated Lie algebra. In fact, (1.4) shows that there is a well defined map $\left[L / L^{k}, L^{k} / L^{k-1}\right] \rightarrow L / L^{k}$ so that $g_{L}^{k}=L^{k} / L^{k+1}$ acts on $L / L^{k}$ (acting trivially on $L^{0} / L^{k}$ ). Exactly the same argument as at the beginning of this section (choosing a complement to $L^{k}$, etc.) gives the existence of $c^{k}$ satisfying the appropriate identities.

Theorem I. Let $L \subset D(V)$ and $M \subset D(W)$ be two transitive closed formal Lie algebras on vector spaces $V$ and $W$ of the same dimension. Let $R(L)$ be the structural order of $L$ and $R(M)$ the structural order of $M$. Then $L$ and $M$ are equivalent if and only if $R(L)=R(M)$ and $\left(L / L^{R(L)}, g_{L}^{R(L)}, c^{R(L)}\right)$ is isomorphic to $\left(M / M^{R(M)}, g_{M}^{R(M)}, c^{R(M)}\right)$.

The proof of Theorem I will be presented in $\$ 7$ after we have discussed the notion of isomorphism of abstract algebras. A few remarks are in order now :

(a) For any $g \subset V \otimes V^{*}$ let us denote by $H^{k, l}(g)$ the groups $H^{k, l}\left(g, g^{(1)}, g^{(2)}, \cdots\right)$. Let us say that $g$ is 3-acyclic if $H^{k 1}(g)=H^{k 2}(g)$ $=0$ for all $k \geqq 1$. A special case of Theorem I would assert that two first order L's with the same acyclic $g$ are equivalent if the corresponding truncated algebras of first order are isomorphic. This is essentially the content of the italicized statement of $\$ 17$ of [3]. The statement there is more general because Cartan considers intransitive groups as well as we have restricted our attention to the transitive groups. Cartan's assumption about the algebra $g$ is that it is "involutive" which is necessary for the application of his existence theorem-the Cartan-Kähler existence theorem (cf. [3], [17] or [18]). For a modern definition of involutiveness, cf. [24] or [27]. For all our present purposes the condition that $g$ be 3 -acyclic suffices. We will therefore not discuss involutiveness here. ${ }^{2}$ The Cartan-Kuranishi prolongation theorem (cf. [18]) which asserts that eventually $g^{k}$ is involutive is replaced by Lemma 4.1. Theorem $\mathrm{I}$ is a version of the statement of

2 It turns out (according to a letter from Serre, cf. Appendix to this paper) that involutiveness is equivalent to $H^{k l}(g)=0$ for all $k$ and $l$. 
$\$ 19$ of [3] (again restricted to the transitive case). Actually that statement corresponds more closely to our Theorem II below.

(b) The hypotheses of Theorem I are frequently very difficult to verify in practice. For instance, let us consider the extreme case where $g=0$ so that $c: V \wedge V \rightarrow V$ actually defines a Lie algebra structure on $V$. The "geometric" content of Theorem I in this case is essentially the assertion that two Lie groups are locally isomorphic if and only if their Lie algebras are isomorphic. While this assertion is not a complete tautology, it isn't very exciting, mainly because no one knows how to classify all Lie algebras up to isomorphism. Of course we do know how to classify some special kinds of Lie algebras (e.g., the compact ones, the semi-simple ones, etc.) and then Theorem I takes on some meaning. It is in this sense that Theorem I solves the problem of classifying transitive geometries.

(c) We should repeat the fact that all we are doing here is completely formal. However Theorem I can be stated as a theorem in geometry and as such has been proved under various additional technical hypotheses (cf. [27, Theorem 3.2 and Theorem 5.3]). The trouble is that in the geometrical case, the proof of Theorem I involves solving partial differential equations and we don't as yet have a suitably general existence theorem. Under the assumptions of analyticity everything goes through nicely (cf. [27, Theorem 3.2]), but the $C^{\infty}$ picture is still incomplete.

5. ${ }^{3}$ So far, we have been discussing the formal version of the problem of what we called "algebraic equivalence" of geometrical structures in the introduction (which in two-dimensional Riemannian geometry corresponds to identifying all spherical geometries). We will now briefly discuss the problem of "geometrical equivalence" (where we distinguish between spheres of different radii). That is what Cartan called the "general equivalence problem" in [4] and [6]. It has been discussed more recently in [2], [9], [13], [15], [20], [27], and [29]. Since this problem has been discussed so extensively from a modern viewpoint in the recent literature, we shall give here only a few indications of the theory; in particular, we shall, for simplicity, restrict attention to structures of (geometrical) first order. In order to motivate the definition we make below, we observe that in order to pick out a specific sphere among all spheres, it suffices to specify the Riemann metric at a single point. More generally, if we know the family of automorphisms, the geometrical structure is specified by a choice at a single point cf. the discussion in $\$ 2$ of [27].

${ }^{3}$ This section has been included only for the sake of completeness. None of the rest of the paper depends on it. 
Let $K^{n}$ be a standard $n$-dimensional vector space and $G l(n, K)$ the group of all nonsingular linear transformations of $K^{n}$. Let $V$ be any $n$-dimensional vector space. We denote by $\mathcal{F}(V)$ the space of bijective linear maps of $K^{n}$ onto $V$. The group $G l(n, K)$ acts in the obvious way on $\mathcal{F}(V)$ : for any $p \in \mathcal{F}(V)$ and any $a \in G l(n, K)$ we set $p a(\xi)$ $=p(a \xi)$ for all $\xi \in K^{n}$. If $W$ is a second vector space and $l$ is a bijective linear map of $V$ onto $W$ then $l$ maps $\mathscr{F}(V) \rightarrow \mathscr{F}(W)$ by setting $l(p)$ $=l \circ p$ for any $p \in \mathcal{F}(V)$. This map obviously commutes with the action of $G l(n, K)$, i.e., $l \circ p a=(l(p)) a$.

Let $(\&$ be a subgroup of $G l(n, K)$. A choice of $p \in F(V)$ gives a representation of \& on $V$. If $q=p a$ where $a \in \&$ then the representation given by $q$ differs from the one given by $p$ by the inner automorphism by $a$. In particular, a choice of orbit of $\mathscr{F}(V)$ under $(\$)$ determines a group, $G$, of linear transformations of $V$. The group $G$ is isomorphic to (f) and the isomorphism is determined up to inner automorphism of $B$.

Definition 5.1. Let \&5 be a connected Lie subgroup of $G l(n, K)$. By a (formal) transitive (S-structure, $B \mathbb{S}(V)$ on $V$ is the pair consisting of an orbit $\mathcal{O}(V)$ of $\mathfrak{F}(V)$ under $\mathbb{B}$, whose associated group is $G$, and a closed transitive $L \subset D(V)$ of geometrical order one such that $g=g_{L}^{0}$ is the Lie algebra of $G$.

Definition 5.2. Let Bos $(V)$ and $B \Theta(W)$ be structures on $V$ and $W$ respectively, where $B_{\mathrm{s}}(V)=(\mathcal{O}(V), L)$ and $B_{\mathrm{O}}(W)=(\mathcal{O}(W), M) . A$ formal power series isomorphism, $\phi$, of $V$ onto $W$ is called a (geometrical) equivalence of $\beta_{\mathbb{S}}(V)$ and $\Theta_{\mathbb{S}}(W)$ if $\phi_{*}(L)=M$ and $l_{\phi}(\mathcal{O}(V))=\mathcal{O}(W)$.

The methods of $\S \S 3$ and 4 can be used to decide when two $\$$ structures are equivalent. Actually, this problem is considerably simpler than that treated in the previous two paragraphs. The reason is that all of the invariants can be brought back to $W^{n}$. Let us illustrate by considering the case where $g$ is 3 -acyclic. (This condition clearly depends only on $\$$.) Let $B_{\mathcal{O}}(V)=(\mathcal{O}(V), L)$ be a $\$$-structure, and let $c \in V \otimes \Lambda^{2}\left(V^{*}\right) / \partial\left(g \otimes V^{*}\right)$ be the structure constant of $L$. Any $p \in \mathcal{O}(V)$ is an isomorphism of $W^{n}$ onto $V$ and as such carries $c$ over into $c \in W^{n} \otimes \Lambda^{2}\left(W^{n *}\right) / \partial\left(\mathfrak{g} \otimes W^{n *}\right)$ where $\mathfrak{g}$ is the Lie algebra of (b); and it is easy to see that this $c$ is independent of the choice of $p \in \mathcal{O}(V)$. We thus get a constant, $c$, lying in a standard space (depending only on (S) as an invariant. The problem of verifying the hypothesis of Theorem I has disappeared: if $\Theta_{\Theta}(W)=(\mathcal{O}(W), M)$ is a second (5)-structure with the same $c$, then the truncated Lie algebra structures on $V$ and $W$ determined by $L$ and $M$ are automatically isomorphic and, in fact, isomorphic by an $l$ with $l(\mathcal{O}(V))=\mathcal{O}(W)$.

6. We now study the "abstract" object whose "representations" 
are the transitive closed $L$ of $\S \S 2-4$. Let $L$ be a Lie algebra and $L^{0}$ a subalgebra of $L$ of finite codimension. We then define $L^{1}$ by

$$
X \in L^{1} \text { if and only if }[X, Y] \in L^{0} \text { for all } Y \in L \text {. }
$$

It is easy to check that $L^{1}$ is an ideal in $L^{0}$. Furthermore, $g_{L}^{0}=L^{0} / L^{1}$ acts in a natural way on $L / L^{0}$. In particular, $L^{1}$ is of finite codimension in $L^{0}$ (and therefore in $L$ ). In general, define $L^{i+1}(i \geqq 0)$ by

(6.2) $X \in L^{i+1}$ if and only if $[X, Y] \in L^{i}$ for all $Y \in L$.

It follows easily from induction and Jacobi's identity that

$$
\left[L^{i}, L^{j}\right] \subset L^{i+j} \text {. }
$$

If we set $g_{L}^{i}=L^{i} / L^{i+1}(i \geqq 1)$ and $g_{L}^{-1}=L / L_{0}$ then we have

$$
\left[\xi_{L}^{k}, \xi_{L}^{l}\right] \subset \xi_{L}^{k+l},
$$

and, in particular

$$
\xi_{L}^{i+1} \subset \dot{g}_{L}^{i(1)}
$$

just like (1.7) and (2.3).

We can define a topology on $L$ by letting the $L_{i}$ be a fundamental system of neighborhoods of 0 . Equation (6.3) implies that the Lie algebra structure is compatible with this topology.

Definition 6.1. An abstract transitive Lie algebra is a Lie algebra, $L$, with a compatible topology such that

(i) $L$ is complete and separated in the (uniform structure associated with) the topology and

(ii) $L$ possesses a subalgebra $L^{0}$ of finite codimension such that the $L^{i}$ defined by (6.2) form a fundamental system of neighborhoods of the origin.

Any subalgebra possessing the properties described in (ii) will be called a fundamental subalgebra.

REMARKs. (a) The fact that $L$ is separated means that for any fundamental subalgebra, $L^{0}$, we have

$$
\bigcap_{i=1}^{\infty} L^{i}=\{0\} .
$$

(b) If $L^{0}$ and $M^{0}$ are fundamental subalgebras then the definition implies that for any $j$ there exist $i$ and $k$ such that

$$
L^{i} \supset M^{j} \supset L^{k} \text {. }
$$

On the other hand, if (6.7) holds, then (6.2) implies that 


$$
L^{i+s} \supset M^{i+s} \supset L^{k+s}
$$

for all integers $s>0$. Thus if $L^{0}$ is a fundamental subalgebra and $M^{0}$ is such that (6.7) holds for some $i, j$, and $k$ then $M^{0}$ is a fundamental subalgebra.

(c) If $L$ is a transitive complete subalgebra of $D(V)$ for some vector space $V$ then $L$, together with the topology induced by $D(V)$ is clearly an abstract transitive Lie algebra.

Definition 6.2. Let $L$ and $M$ be abstract transitive Lie algebras and let $\phi: L \rightarrow M$. We say that $\phi$ is a homomorphism if $\phi$ is continuous and is a homomorphism of the underlying Lie algebra structures. We say that $\phi$ is an isomorphism if it is a homeomorphism and an isomorphism of the Lie algebra structures. An isomorphism of $L$ with a complete transitive $L \subset D(V)$ is called a realization of $L$.

Observe that any realization of $L$ picks out a distinguished $L^{0}$.

Definition 6.3. Let $L$ be an abstract transitive Lie algebra. A fundamental subalgebra $L^{0}$ is called regular if

$$
H^{k l}\left(L^{0} / L^{1}, L^{1} / L^{2}, \cdots\right)=0 \quad \text { for } l=1,2 \text { and } 3 \text {, and } k>0 .
$$

In particular, if $L^{0}$ is a regular fundamental subalgebra then

$$
L^{i+1} / L^{i+2}=\left(L^{i} / L^{i+1}\right)^{(1)} .
$$

According to Lemma 4.1 and remark (b) above, $L^{k}$ is a regular subalgebra for sufficiently large $k$. Thus every $L$ possesses a regular subalgebra.

Just as in $\$ 4$, the Lie algebra structure of $L$ induces a truncated Lie algebra structure on $L / L^{0}$. We can now state the main theorems of our subject.

Theorem II (Existence and Uniqueness Theorem). Let $(V, g, c)$ be a truncated Lie algebra structure with $H^{k 1}(g)=H^{k 2}(g)$ $=H^{k 3}(g)=0$ for all $k \geqq 1$. Then there exists an abstract transitive Lie algebra $L$, with a regular fundamental subalgebra $L_{0}$, such that the truncated Lie algebra structure induced on $L / L^{0}$ is isomorphic to $(V, g, c)$. Furthermore, if $M, M^{0}$ is a second such abstract transitive Lie algebra and regular subalgebra then there exists an isomorphism, $\phi$, of $L$ onto $M$ carrying $L^{0}$ into $M^{0}$. In particular, two abstract Lie algebras, $L$ and $M$ are isomorphic, if and only if they possess regular fundamental subalgebras, $L^{0}$ and $M^{0}$ such that the truncated Lie algebra structures induced in $L / L^{0}$ and $M / M^{0}$ are isomorphic.

TheOREM III (REAlization TheOREM). Let $L$ be an abstract transitive Lie algebra and $L^{0}$ a fundamental subalgebra. Then $L$ is isomorphic to a transitive closed subalgebra, $L$, of $D\left(L / L^{0}\right)$. Furthermore, $L$ is determined up to equivalence. 
Notice that Theorems II and III together imply Theorem I. In fact, under the hypotheses of Theorem I, Theorem II assures that $L$ and $M$ are isomorphic as abstract algebras and Theorem III implies that the represented algebras are equivalent. The proofs of Theorems II and III will be presented in the next sections.

Let $L \subset D(V)$ and $M \subset D(W)$ be transitive closed subalgebras (where $V$ and $W$ may be of differing dimensions). Suppose that $L$ and $M$ are isomorphic as abstract algebras. Then Theorem III implies that we can, by choosing small enough fundamental subalgebras, find realizations of $L$ and $M$ which are equivalent. Geometrically, choosing a "smaller" subalgebra amounts to realizing the abstract algebra on a "bigger" space. If $L$ is already given as a realized algebra, $L$, then the "bigger" realization can be called a "prolongation" of $L$. This accounts for Cartan's definition in $\$ 18$ of [3], that "two groups are called isomorphic if they possess prolongations which are equivalent."

The uniqueness part of Theorem II corresponds to the italicized statement in $\$ 19$ of [3] and the "second fundamental theorem" in [7]. The existence part corresponds to the statement of $\$ 24$ in [3] and the "third fundamental theorem" in [7]. Again, our versions are restricted to the transitive case whereas Cartan considers the "intransitive groups" as well.

7. We now proceed to the proof of Theorem II. Let $(V, g, c)$ be a truncated Lie algebra. We let $g^{(1)}\left(\subset g \otimes V^{*}\right)$ act on $V+g$ by acting trivially on $g$. In this way $g^{(1)}$ can be regarded as an abelian Lie algebra. We say that a truncated Lie algebra $\left(V+g, g^{(1)}, c^{1}\right)$ is an extension of $(V, g, c)$ if we can find representatives $\tilde{c}$ of $c$ and $\tilde{c}^{1}$ of $c^{1}$ so that

$$
\begin{aligned}
& \tilde{c}^{1}(u \wedge v)=\tilde{c}(u \wedge v) \bmod g \quad \text { for all } u, v \in V, \\
& \tilde{c}^{1}(X \wedge v)=X \cdot v \bmod g \quad \text { for all } X \in g \text { and } v \in V,
\end{aligned}
$$

and

$$
\tilde{c}^{1}(X \wedge Y)=[X, Y] \quad \text { for } X, Y \in g .
$$

Let $g$ be a subspace of $V \otimes V^{*}$. We say that $V$ acts trivially on a subspace $V_{0}$ of $V$ if $X v=0$ for all $X \in g$ and $v \in V_{0}$. We denote by $g \mid V / V_{0}$ the induced space of linear transformations of $V / V_{0}$.

LEMma 7.1. Let $g \subset V \otimes V^{*}$ act trivially on $V_{0} \subset V$ and satisfy $H^{k l}\left(g \mid V / V_{0}\right)=0$ for some $k$ and all $1 \leqq l \leqq n$. Then $H^{k l}(g)=0$ for the same range of $k$ and $l$. 
Proor. It follows immediately from the definitions that $X v_{0}=0$ for any $X \in g^{(k)}$ and $v_{0} \in V_{0}$. Our proof of the lemma will therefore be the same for all $k$ and will be by induction on $n$. For $n=1$, let $\xi \in \operatorname{Hom}\left(V, g^{(k)}\right)$ be a cycle, i.e., satisfying

$$
\xi(u) v-\xi(v) u=0 .
$$

If we take $u \in V_{0}$ in this equation we see that $\xi(u)=0$ for all $u \in V_{0}$, i.e., $\xi$ defines an element $\tilde{\xi}$ of $\operatorname{Hom}\left(V / V_{0}, g^{(k)}\right)$ which is clearly a cycle. We can write $\tilde{\xi}=\partial \tilde{\eta}$ where $\tilde{\eta} \in g^{(k+1)}$. We then have $\xi(u)=\eta \cdot u$ and are done. Now assume the lemma for the case $n-1$. Let $\xi \in g^{(k)} \otimes \Lambda^{n}\left(V^{*}\right)$ be a cycle, i.e., satisfying

$$
\sum(-1)^{i} \xi\left(v_{1}, \cdots, \hat{v}_{i}, \cdots, v_{n+1}\right) v_{i}=0 .
$$

Since $g^{(k)}$ acts trivially on $V_{0}$, if we fix $v_{n+1} \in V_{0}$ then $\xi\left(\cdots, v_{n+1}\right)$ $\in g^{(k)} \otimes \Lambda^{n-1}\left(V^{*}\right)$ is a cycle. We therefore can write

$$
\xi\left(\cdots, v_{n+1}\right)=\partial \eta\left(v_{n+1}\right)
$$

where $\eta\left(v_{n+1}\right) \in g^{(k+1)} \otimes \Lambda^{n-2}\left(V^{*}\right)$. Here $\eta(\cdot)$ can be regarded as an element of $\operatorname{Hom}\left(V_{0}, g^{k+1} \otimes \Lambda^{n-2}\left(V^{*}\right)\right)$. Extend $\eta$ to be defined on all of $V$ so as to get an element of $\operatorname{Hom}\left(V, g^{(k+1)} \otimes \Lambda^{n-2}\left(V^{*}\right)\right)$ $=g^{(k+1)} \otimes \Lambda^{n-2}\left(V^{*}\right) \otimes V^{*}$ and anti-symmetrize so as to obtain an element of $g^{(k+1)} \otimes \Lambda^{n-1}\left(V^{*}\right)$ which we shall continue to denote by $\eta$. Then $\xi-\partial \eta$ is a cycle which vanishes if any one of its arguments lies in $V_{0}$. It therefore defines a cycle of $g^{(k)} \otimes \Lambda^{n}\left(\left(V / V_{0}\right)^{*}\right)$, and therefore is a coboundary, as in the case $n=1$.

Lemma 7.2. Let $(V, g, c)$ be a truncated Lie algebra, and let $\phi: V \rightarrow V$ be a nonsingular linear transformation satisfying $\phi X u=X u=X \phi u$ for all $X \in g$ and $u \in V$. Let $\tilde{c}$ be a representative of $c$. Let $\phi^{*} \tilde{c} \in \operatorname{Hom}(V \wedge V, V)$ be defined by $\phi^{*} \tilde{c}(u \wedge v)=\phi \tilde{c}\left(\phi^{-1} u \wedge \phi^{-1} v\right)$. Then $\phi^{*} \tilde{c}$ defines a truncated Lie algebra structure $\left(V, g, \phi^{*}\right)$ on $V$ (relative to $g$ ). This structure is independent of the choice of $\tilde{c}$ and $\phi$ is an isomorphism of $(V, g, c)$ with $\left(V, g, \phi^{*} c\right)$.

The proof of this lemma is obvious from the definitions.

Lemma 7.3. Let $(V, g, c)$ be a truncated Lie algebra where $g$ acts trivially on the subspace $V_{0}$. Suppose $\tilde{c}\left(V_{0} \wedge V_{0}\right) \subset V_{0}$ for some (and hence all) representatives of $c$ and that $H^{k i}\left(\left.g\right|_{V / V_{0}}\right)=0$ for $i=1,2,3$. Then there exists an extension $\left(V+g, g^{(1)}, c\right)$ of $(V, g, c)$ with $\tilde{c}^{1}\left(V_{0}+g \wedge V_{0}+g\right) \subset V_{0}+g$. Any two such extensions are isomorphic.

Proof. Let $\tilde{c}$ be a fixed representative of $c$. We would like to find a $b \in g \otimes \Lambda^{2}\left(V^{*}\right)=\operatorname{Hom}(V \wedge V, g)$ and an $S \in \operatorname{Hom}(g \otimes V, g)$ such that if we set 


$$
\begin{aligned}
\tilde{z}^{1}(u \wedge v) & =\tilde{c}(u \wedge v)+b(u \wedge v), \\
\tilde{c}^{1}(X \wedge v) & =X v+S(X \otimes v),
\end{aligned}
$$

and

$$
\tilde{c}^{1}(X \wedge Y)=[X, Y]
$$

then $\tilde{z}^{1}$ defines a truncated Lie algebra structure on $V+g$. In other words we wish to be able to find a $b^{1} \in \operatorname{Hom}\left((V+g) \wedge(V+g), g^{(1)}\right)$ and an $S^{1} \in \operatorname{Hom}\left(g^{(1)} \otimes(V+g), g^{(1)}\right)$ so that

$$
\left(\tilde{c}^{1}\right)^{2}+\partial b^{1}=0
$$

and

(7.8) $T \tilde{c}^{1}(w \wedge z)-\tilde{c}^{1}(T w \wedge z)-\tilde{c}^{1}(w \wedge T z)=S^{1}(T \otimes w) z-S^{1}(T \otimes z) w$

for all $T \in g^{(1)}, w$ and $z \in V+g$. Now by the definition of $g^{(1)}$ we have $\partial b^{1}\left(w_{1} \wedge w_{2} \wedge w_{3}\right) \in g$ for any $w_{1}, w_{2}, w_{3} \in V+g$. Thus the $V$ component of $(\tilde{c})^{2}$ must vanish by (7.7). If we apply this to $v_{1}, v_{2}, v_{3} \in V$ and compare with (7.4) we see that

$$
\partial b+\tilde{c}^{2}=0 .
$$

Such a $b$ exists by assumption since $(V, g, c)$ is a truncated Lie algebra. It is determined up to a cycle. According to Lemma 7.1, $H^{1,2}(g)=0$ so that $b$ is determined by (7.9) up to the addition of a term $\partial d$ for some $d \in \operatorname{Hom}\left(V, g^{(1)}\right)$.

If we now look at the $V$ component of $\left(c^{1}\right)^{2}$ applied to $X \in g, u$ and $v \in V$ we see that $S$ must satisfy

(7.10) $X c(u \wedge v)-c(X u \wedge v)-c(u \wedge X v)-S(X \otimes u) v+S(X \otimes v) u=0$.

Again such an $S$ exists by assumption. It is determined up to adding a term $T \in \operatorname{Hom}(g \otimes V, g)$ where $T \in \operatorname{Hom}\left(g, g^{(1)}\right)$. Let us define $d^{1} \in \operatorname{Hom}\left(V+g, g^{(1)}\right)$ by setting

$$
d^{1}(v)=d(v), \quad d^{1}(g)=T(g) .
$$

Then we see that $\tilde{c}^{1}$ is determined up to $\partial d^{1}$ (where now $\left.\partial: \operatorname{Hom}\left(V+g, g^{(1)}\right) \rightarrow(V+g) \otimes \Lambda^{2}\left((V+g)^{*}\right)\right)$. Thus if there exists a $\tilde{c}^{1}$ satisfying (7.4)-(7.8) it defines a unique truncated Lie algebra structure on $V+g$, given by (7.7) and (7.10).

We now must check that (7.9) and (7.10) do indeed define a truncated Lie algebra structure, i.e., that there exist $b^{1}$ and $S^{1}$ so that (7.7) and (7.8) are satisfied. In view of Lemma 7.1 applied to $V+g$ and $g^{(1)}$ and the fact that $H^{2 i}(g)=0(i=1,2,3)$ it suffices to prove that the $g$ component of $\left(\tilde{c}^{1}\right)^{2}$ is a cycle (as an element of 
$\left.g \otimes \Lambda^{3}\left(V^{*}\right)\right)$ and that the $g$ component of $c_{T}^{1}$ is a cycle in $g \otimes \Lambda^{2}\left(V^{*}\right)$, where $c_{T}^{1}(w \wedge z)=T c^{1}(w \wedge z)-c^{1}(T w \wedge z)-c^{1}(w \wedge T z)$. These facts follow from repeated application of (7.9) and (7.10) and will be left as straightforward (if rather tedious) verification for the reader. We have thus proved the existence part of the lemma. We have also seen that $c^{1}$ is uniquely determined by (7.4)-(7.6) once we have made a choice of $z$. Suppose we have a second extension $\left(V+g, g^{(1)}, c^{1 /}\right)$ of $(V, g, c)$ corresponding to a choice $\hat{c}$ where

$$
\hat{c}(u \wedge v)-\tilde{c}(u \wedge v)=S(u) v-S(v) u
$$

where $S \in \operatorname{Hom}(V, g)$. Let $\phi: V+g \rightarrow V+g$ be defined by

$$
\begin{aligned}
& \phi(u)=u+S(u) \quad \text { for } u \in V \text {, } \\
& \phi(X)=X \quad \text { for } X \in g \text {. }
\end{aligned}
$$

Then $\phi$ satisfies the hypothesis of Lemma 7.2 and $\phi^{*} \tilde{c}^{1 \prime}$ satisfies (7.4)(7.6) for some representative $\tilde{c}^{1 \prime}$ of $c^{1}$. This proves that any two extensions are isomorphic, completing the proof of the lemma.

It should be remarked that the uniqueness part of the lemma depends only on the vanishing of $H^{k 1}$ and $H^{k 2}$ while we used $H^{k z}=0$ for the existence part.

We can now apply Lemma 7.3 again, replacing $V$ by $V+g, g$ by $g^{(1)}$ and $c$ by $c^{1}$. Repeated application of the lemma gives a truncated Lie algebra $\left(V+g+\cdots+g^{(n)}, g^{(n+1)}, c^{n+1}\right)$ determined up to isomorphism. Passing to the limit we obtain an abstract transitive Lie algebra structure on $L=\lim \left(V+g+g^{(1)}+\cdots\right)$ determined up to isomorphism. This proves Theorem II.

8. We now turn to the proof of Theorem III. Let $L$ be an abstract transitive Lie algebra and $L_{0}$ a fundamental subalgebra. Let us set $V=L / L^{0}$. We wish to find a realization of $L$ as a transitive subalgebra of $D(V)$ where $L_{0}$ becomes the subalgebra of vector fields vanishing at the origin. It will be convenient to prove the existence and the uniqueness of the realization simultaneously. We wish to find a mapping of $L \rightarrow D(V)$ sending $\mathrm{x} \rightarrow x \in D(V)$ where we shall write the Taylor series expansion of $x$ as

$$
x=\mathrm{x}_{0}+\mathrm{x}_{1}+\cdots+\mathrm{x}_{\boldsymbol{i}}+\cdots
$$

so that $\mathbf{x}_{i} \in V \otimes S^{i}\left(V^{*}\right)$. We shall determine the $\mathbf{x}_{i}$ inductively. By definition, we have

$$
\mathrm{x}_{0}=\mathrm{x} / L^{0} \in V
$$

so that $x_{0}$ is uniquely determined. We now wish to choose $x_{1} \in V \otimes V^{*}$ so that 


$$
x_{1} y_{0}-y_{1} x_{0}=[x, y]_{0} .
$$

Notice that for $x \in L_{0}$, this defines $x_{1}$ uniquely since $x_{0}=0$. Let us choose a complement $V$ to $L^{0}$ which we shall hold fixed throughout the following discussion. To define $\mathrm{x}_{1}$ for all $\mathrm{x} \in L$ it suffices to define it for $x \in V$. Equation (8.3) then says that we are given an element, $\tilde{c}$, of $V \otimes \Lambda^{2}\left(V^{*}\right)$ (the Lie bracket on $V$ regarded as a map of $V \wedge V \rightarrow V)$ and wish to find an element $\tilde{d}$ of $\operatorname{Hom}\left(V, V \otimes V^{*}\right)$ $=V \otimes V^{*} \otimes V^{*}$ with $\partial \tilde{d}=\tilde{c}$. This we can always do. The element $\tilde{d}$ is determined up to cycle, i.e., an element of $V \otimes S^{2}\left(V^{*}\right)$. In other words, if $L \rightarrow L^{\prime}$ sending $\mathrm{x} \rightarrow x^{\prime}$ with

$$
x^{\prime}=\mathrm{x}_{0}+\mathrm{x}_{1}+\cdots
$$

is a second realization of $L$ on $V$ we must have

$$
\mathbf{x}_{1}^{\prime}=\mathrm{x}_{1}+\left\langle\mathrm{x}_{0}, \psi_{2}\right\rangle
$$

where $\psi_{2} \in V \otimes S^{2}\left(V^{*}\right)$ and \langle\rangle is the pairing of $V \otimes\left(V \otimes S^{2}\left(V^{*}\right)\right)$ $\rightarrow V \otimes V^{*}$. If we consider a formal power series isomorphism $\psi^{2}$ given by

$$
\psi^{2}=i d+\psi_{2}+\text { higher order terms }
$$

then (8.5) says that $\psi_{*}^{2}(x)=x^{\prime}$ up through the term of first order.

Now we shall proceed by induction. For any $w \in D(V)$ we shall denote by $w_{k}$ the component of $w$ of degree $k$ (lying in $V \otimes S^{k}\left(V^{*}\right)$ ) while we shall denote by $\{w\}_{k}$ the sum of terms of order up to $k$ so that

$$
\{w\}_{k}=w_{0}+\cdots+w_{k} .
$$

Lemma 8.1. Suppose that we have a map of $L \rightarrow D(V)$ sending $\mathrm{x} \rightarrow\{x\}_{k}=\mathrm{x}_{0}+\cdots+\mathrm{x}_{k}$ such that

$$
\{[x, y]\}_{k-1}=\left\{\left[\{x\}_{k},\{y\}_{k}\right]\right\}_{k-1} \text {. }
$$

We can then find a map $L \rightarrow V \otimes S^{k+1}\left(V^{*}\right)$ given by $\mathrm{x} \rightarrow \mathbf{x}_{k+1}$ so that $\{x\}_{k+1}=\{x\}_{k}+\mathbf{x}_{k+1}$ satisfies

$$
\{[x, y]\}_{k}=\left\{\left[\{x\}_{k+1},\{y\}_{k+1}\right]\right\}_{k} .
$$

If $\mathbf{x}_{k+1}^{\prime}$ is second such choice with $\left\{x^{\prime}\right\}_{k+1}=\{x\}_{k}+\mathbf{x}_{k+1}^{\prime}$ then there is a formal power series map $\psi^{k+1}$ which is the identity up through terms of order $k$ such that

$$
\left\{\psi_{*}^{k+1}\{x\}_{k+1}\right\}_{k+1}=\left\{x^{\prime}\right\}_{k+1}
$$

(In the left-hand side of (8.6) we should perhaps write $\left\{\{[x, y]\}_{k}\right\}_{k-1}$ 
but we have used an obvious shorter notation; the same goes for (8.7).)

It is clear that a repeated application of Lemma 8.1 and passage to the limit implies Theorem III.

Proof of Lemma 8.1. In view of (8.6), in order for (8.7) to hold we must merely check the terms of order $k$. We must therefore have

$$
\mathbf{x}_{k+1} y_{0}-y_{k+1} \mathbf{x}_{0}=-\left[\{x\}_{k},\{y\}_{k}\right]_{k}+[x, y]_{k} .
$$

For $x \in L_{0}$ this defines $x_{k+1}$ uniquely as an element of $\operatorname{Hom}\left(V, V \otimes S^{k}\left(V^{*}\right)\right)=V \otimes S^{k}\left(V^{*}\right) \otimes V^{*}$. We must check that it actually lies in $V \otimes S^{k+1}\left(V^{*}\right)$.

For $x$ and $y$ in $V$ the right-hand side of (8.8) can be regarded as an element of $V \otimes S^{k}\left(V^{*}\right) \otimes V^{*} \wedge V^{*}$. We would like to verify that it is a cycle.

Both of these verifications would follow if we know that

$$
\begin{aligned}
{\left[\{x\}_{k},\{y\}_{k}\right]_{k} z_{0}+\left[\{y\}_{k},\{z\}_{k}\right]_{k} x_{0}+\left[\{z\}_{k},\{z\}_{k}\right]_{k} y_{0} } \\
=[x, y]_{k} z_{0}+[y, z]_{k} x_{0}+[z, x]_{k} y_{0} .
\end{aligned}
$$

Let $\sigma$ denote complete anti-symmetrization with respect to $x, y, z$. Then by the Jacobi identity for $D(V)$ we have

$$
\left.\sigma\left(\left[\{x\}_{k},\{y\}_{k}\right],\{z\}_{k}\right]\right)=0
$$

so that in particular

$$
\left.\sigma\left(\left[\{x\}_{k},\{y\}_{k}\right],\{z\}_{k}\right]_{k-1}\right)=0 .
$$

But

(8.10) $\left.\left[\{x\}_{k},\{y\}_{k}\right],\{x\}_{k}\right]_{k-1}=\left[\{x\}_{k},\{y\}_{k}\right] z_{0}+\left[\left\{\left[\{x\}_{k},\{y\}_{k}\right]\right\}_{k-1},\{z\}_{k}\right]_{k-1}$.

Now by induction we have

$$
\left\{\left[\{x\}_{k},\{y\}_{k}\right]\right\}_{k-1}=\{[x, y]\}_{k-1}
$$

so that

$$
\left[\left\{\left[\{x\}_{k},\{y\}_{k}\right]\right\}_{k-1},\{z\}_{k}\right]_{k-1}=[[x, y], z]_{k-1}-\left[[x, y]_{k}, z_{0}\right] .
$$

Substituting into (8.10), applying $\sigma$ and using the Jacobi identity on $L$ we conclude (8.9). This proves the existence part of the lemma. The uniqueness follows just as in the case $k=0$ since $\mathbf{x}_{k+1}$ is determined up to a coboundary. We have thus proved Theorem III.

Notice that for the case $L^{0}=0$ (so that $L$ is an abstract finite dimensional Lie algebra) Theorem III is just the formal analogue of the classical "third fundamental theorem" of the theory of Lie groups 
which asserts that every Lie algebra is the Lie algebra of some Lie group.

9. We will now attempt to present a brief discussion of the literature surrounding our subject. The fundamental papers on the subject are, of course, those of Lie [22] and Cartan [3]-[7]. For a different presentation of the foundations of the infinite groups (including the intransitive ones) see Kuranishi [18] and [19]. Kuranishi is perhaps closer, in spirit, to the ideas and language of Cartan and bases his presentation on the powerful Cartan-Kuranishi prolongation theorem. His theory, like Cartan's is valid only in the analytic case. For a more "geometrical" presentation using the infinitesimal approach, cf. [27]. We now turn to more specific points:

(a) Solving the partial differential equations. As we have emphasized several times the theory we have presented here is purely formal. There are two corresponding geometrical theories-where the data are always analytic or where the data are $C^{\infty}$. It is the analytic case that Cartan treats and uses as his main tool the existence theorem he developed for this purpose- the Cartan-Kähler existence theorem (cf. [1], [3] and [17]). (Reference [1] provides an exposition of Cartan's papers [3]-[6], adhering rather closely to the original.) Actually, the methods we present here can be used in the analytic case. Such a treatment will be presented elsewhere. In the $C^{\infty}$ case the situation is not yet in good shape on account of the lack of existence theorems like the one in [25] which are needed, cf. [27] and the comments at the end of $\S 6$, above.

(b) Classification of the simple groups. Let us call an abstract transitive algebra simple if it admits no closed ideal. The classification of the simple finite (dimensional) groups (in the real and complex) case is well known. In [5], Cartan purports to give a classification of the transitive simple infinite groups in the complex case. His main idea is to classify the "primitive" represented infinite "groups." (A primitive group is one which does not leave invariant a completely integrable differential system. In our formal model, the corresponding notion turns out to be a transitive closed algebra $L \subset D(V)$ with no proper subalgebras strictly containing $L_{0}$.) Every simple group can be represented as a primitive group of transformations. In fact, let $L^{\prime \prime}$ be a maximal subalgebra of finite codimension. Then $\cap L^{\prime i}$ is a closed ideal and hence $\cap L^{\prime i}=0$. By the analogue of a theorem of Chevalley (p. 270 of [31]) we conclude that $L^{\prime 0}$ is a fundamental subalgebra, so $L / L^{\prime 0}$ gives a primitive representation. Cartan then proceeds to classify the primitive infinite groups. For this he first classifies (Chapter V of [5]) all (complex) irreducible Lie 
algebras $g \subset V \otimes V^{*}$ for which $g^{(2)} \neq 0$. (He calls "semi-involutive" those $g$ with $g^{(k)} \neq 0$ for all $k$; i.e., the $g$ that we call of "infinite type.") He gives very few details of this classification. For more details cf. Chapter V of [27]. Both the procedures in [5] and in [27] are messy but the results seem to be correct. (For the extension of the classification to the real case cf. [24].) In Cartan's application of this classification to the classification of the primitive group there seems to be a serious gap (in the proof of Theorem II of $\S$ ), cf. the discussion in $\$ 2.11$ of [27]. As a first step towards filling in this gap, cf. [16]. A classification of the irreducible infinite groups (i.e., those which don't have invariant any nontrivial differential system (integrable or not)) is given in Chapter V of [27].

(c) The intransitive situation, geometry. There are two possible generalizations of our subject to the intransitive case-the study of intransitive geometries and the study of the intransitive groups. Most differential geometrical structures don't admit any nontrivial automorphisms so that they can't be studied by studying their groups of automorphisms. Nevertheless the methods of the "general (geometrical) equivalence problem" apply and, in fact, give all the crucial invariants. (The geometrical equivalence problem is not to be formulated as we presented it here in $\$ 5$. The presentation of $\$ 5$ was artificial and just constructed so as to fit into our framework here.) The constant invariants of the transitive case (cf. \$5) become functions in the general case; functions which are defined on suitable spaces associated with the geometry. (For example, the only nonvanishing Spencer homology group for $g=o(n)$ (the orthogonal algebra) is $H^{21}$. An element of $H^{21}$ corresponds to the "curvature." In general the curvature of a Riemann manifold is a function.) For a treatment of the general equivalence problem, cf. [2], [6], [9], [12], [15], [20], [27, Chapter II] and [29, Chapter VII]. In [15] many of the "tensors" of classical differential geometries (such as conformal differential geometry, projective differential geometry, etc.) are shown to be the appropriate specialization of the "structure functions" of the general equivalence problem.

(d) The intransitive situation, algebra. The intransitive "groups" were studied by Cartan along with the transitive ones, cf. [3] and [4] and Kuranishi's presentation [19]. The paper [4] is of fundamental importance. We hope to present a formal version of the intransitive groups, generalizing the methods of this paper, in the near future. The study of the intransitive groups may be of importance in physics. The various "gauge groups" [30] are examples of intransitive infinite groups. 


\section{APPENDIX ${ }^{4}$}

Let $g$ be a subspace of $\operatorname{Hom}(V, W)$. The prolongations of $g, g^{(1)}$, $g^{(2)}$ etc. will be defined as above. Let $H$ be a subspace of $V$. We will denote by $g_{H}$ the subspace of $g$ consisting of all mappings which are identically zero on $H$. Let $\rho_{k}$ be the minimum of $\operatorname{dim} g_{H}$ where $H$ runs through the set of $k$-dimensional subspaces of $V$.

One can easily establish the following result (cf. [23] or [27])

$$
\operatorname{dim} g^{(1)} \leqq \rho_{0}+\rho_{1}+\cdots \rho_{k-1} .
$$

If there is equality in (1.1) then the space $g$ is called involutive.

A simple criterion for involutiveness is the following:

There exists a basis $t_{1}, \cdots, t_{n}$ for $V$, such that if $H_{i}$ is the space spanned by $t_{1}, \cdots, t_{n}$ then the mapping

$$
\lambda\left(t_{i+1}\right)\left(g_{H_{i}}\right)^{(1)} \rightarrow g_{H_{i}}, \quad s \rightarrow s_{t_{i+1}},
$$

is surjective.

To prove the equivalence one way is easy. We simply note that the kernel of $\lambda\left(t_{i+1}\right)$ is $\left(g_{H_{i+1}}\right)^{(1)}$, and therefore $\operatorname{dim}\left(g_{H_{i}}\right)^{(1)}=\operatorname{dim} g_{H_{i}}$ $+\operatorname{dim}\left(g_{H_{i+1}}\right)^{(1)}$ so by induction: $\operatorname{dim} g^{(1)}=\operatorname{dim} g+\operatorname{dim} g_{H_{1}}+\ldots$ $+\operatorname{dim} g_{H_{n-1}}$. The converse is a little more difficult, and we will not bother to prove it here, citing [27] as a reference.

A basis for $V$ satisfying the conditions above will be called quasiregular. In [25] or [27] it is proved that

If $t_{1}, \cdots, t_{n}$ is quasi-regular for $g$ it is also quasi-regular for $g^{(1)}\left(g^{(1)}\right.$ regarded as a subspace of $\left.\operatorname{Hom}(V, g)\right)$.

In particular if $g$ is involutive $g^{(1)}$ is involutive.

Let $E^{k}$ be the vector space dual to $g^{(k-1)}$. By convention we will set $E^{0}=V$. If $t$ is an element of $V$ it gives a mapping $g^{(k)} \rightarrow g^{(k-1)}$, sending $s \rightarrow s_{t}$, and therefore a dual mapping $E^{k} \rightarrow E^{k+1}$. If $s$ is an element of $E^{k}$ we will denote its image by $t s$.

By the definition of $g^{(k)}, t_{1}\left(t_{1} s\right)=t_{2}\left(t_{1} s\right)$ for $s$ in $E^{k}$ and $t_{1}, t_{2}$ in $V$. Therefore the mapping $V \otimes E^{k} \rightarrow E^{k+1}$ we have just defined extends to a mapping of $S(V) \otimes \sum E^{k}$ into $\sum E^{k}$. This defines an $S(V)$ module structure on $\sum E^{k}$. Moreover $E=\sum E^{k}$ is a homomorphic image of the $S(V)$-module $W^{*} \otimes S(V)$; therefore it is Noetherian. Furthermore the Spencer homology defined above dualizes to the Koszul homology of $E$.

Let $t_{1}, \cdots, t_{k}$ be a set of linearly independent elements in $V$ and $H$ the vector space they span The annihilator of $g_{H}^{(k)}$ in $E^{k+1}$ is $\left(t_{1}, \cdots, t_{k}\right) E^{k}$; so the dual of $g_{H}^{(k)}$ is identical with $E^{k+1} /\left(t_{1}, \cdots, t_{k}\right) E^{k}$.

\footnotetext{
${ }^{4}$ Added in proof.
} 
Assume now that $g$ is involutive. The definition of involutiveness translated into its dual form says: There exists a basis, $t_{1}, \cdots, t_{n}$, of $V$ such that

is $1-1$.

$$
t_{k+1}\left(E^{k} /\left(t_{1}, \cdots, t_{k}\right) E^{k-1}\right) \rightarrow E^{k+1} /\left(t_{1}, \cdots, t_{k}\right) E^{k}
$$

In other words, letting $E^{+}$denote the sum $\sum_{k>0} E, g$ is involutive if and only if there exists a sequence $t_{1}, \cdots, t_{n}$ in $V$ such that no nonzero elements of $E^{+} /\left(t_{1}, \cdots, t_{k}\right) E$ is annihilated by $t_{k+1}$.

The following letter from Serre (reproduced with his kind permission) clears up the relation between the vanishing of the $H^{k i}(g)$ (used for $k=1,2,3$ above) and involutiveness (used by Cartan).

Paris, 17 Juillet 1963

Votre traduction, en termes de modules, de la notion d'algèbre involutive est tout à fait satisfaisante. Il est bien vrai en tout cas que "involutif" équivaut à dire que certains groupes d'homologie sont nuls, comme vous le conjectures. La démonstration consiste à reprendre celle du cas local, en faisant attention aux degrés (le cas local est traité dans Auslander-Buchsbaum, ainsi que dans mon papier au symposium Tokyo-Nikko-cf. notamment $\$ 3$ de ce papier).

Voici un peu plus de détails:

Soit $S=k\left[X_{1}, \cdots, X_{n}\right]$ l'algèbre de polynômes sur un corps $k$ (supposé infini, mais pas nécessairement de caractéristique zéro). On note $I$ l'idéal maximal $\left(X_{1}, \cdots, X_{n}\right) ; S / I=k$. On considère des $S$ modules gradués de type fini $E=\sum_{k \geq 0} E^{k}$, les degrés $k$ prenant des valeurs $\geqq 0$. On note $E^{+}$la somme directe des $E^{k}$ pour $k \geqq 1$; lorsque $E$ est engendré par ses éléments de degré zéro (c'est le cas pour le module que vous étudiez), on a $E^{+}=I E$.

Si $E$ est un tel module, on définit comme vous savez le complexe de l'algèbre extérieure $K(X, E)$ associé à $E$ et à la suite des $X_{i}$. La graduation de $E$ définit une graduation de $K(X, E)$. Les groupes d'homologie correspondants sont notés $H_{i}(E)$; eux aussi sont gradués à degrés $\geqq 0$, ce qui donne un sens à l'expression $H_{i}(E)^{+}$. Noter que $H_{n}(E)$ s'identifie au sous-espace vectoriel de $E$ formé des éléments annulés par $I$.

Lemme 1. Soit $E=\sum_{k \geq 0} E^{k}$ comme ci-dessus. Les conditions suivantes sont équivalentes:

(a) $H_{n}(E)^{+}=0$.

(b) $I x=0, x \in E$ entraine $x \in E^{0}$.

(c) Il existe une combinaison linéaire $t$ des $X_{i}$ telle que $t x=0, x \in E$ entraîne $x \in E^{0}$. 
(d) Pour toute combinaison linéaire $t$ des $X_{\mathbf{i}}$ n'appartenant pas d un nombre fini de sous-espaces vectoriels, la condition $t x=0, x \in E$ entraîne $x \in E^{0}$.

(Au lieu de d), je dirai comme vous "pour tout $t$ générique $\cdots .$. .)

(a) $\Leftrightarrow$ (b) est trivial. Idem pour $(d) \Rightarrow(c) \Rightarrow(b)$. Reste à voir que (b) $\Rightarrow$ (d). Soit $H$ le sous-espace de $E$ formé des éléments $x$ tels que $I x=0$. On a $H \subset E^{0}$; soit $K$ un supplémentaire de $H$ dans $E^{0}$, et posons $F=K \oplus E^{1} \oplus E^{2} \oplus \cdots$. On a $E=H \oplus F$. Il résulte de (b) qu'aucun élément non nul de $F$ n'est annulé par $I$. Il en résulte que $I$ n'est pas un ideal premier associé à $F$ (au sens de Bourbaki, Alg. Comm., Chap. IV, $\S 1)$; soient $p_{1}, \cdots, p_{r}$ les dits idéaux premiers associés. Si l'on choisit $t \notin p_{1} \cup \ldots \cup \cup_{p_{r}}$, on sait que $t$ est non diviseur de zéro dans $F$, ce qui démontre (d).

Lemme 2. Soient E et $t$ vérifiant les conditions de (c) ci-dessus. Pour tout $i$, on a une suite exacte:

$$
0 \rightarrow H_{i}(E)^{+} \rightarrow H_{i}(E / t E)^{+} \rightarrow H_{i-1}(E)^{+} \rightarrow 0 .
$$

On écrit comme ci-dessus $E=H \oplus F$. On remarque que $H_{i}(E)^{+}$ $=H_{i}(H)^{+} \oplus H_{i}(F)^{+}=H_{i}(F)^{+}$, et de même $H_{i}(E / t E)^{+}=H_{i}(F / t F)^{+}$. On peut donc remplacer $E$ par $F$ sans rien changer; cela revient à supposer que $H=0$. La multiplication par $t$ est alors injective; en effet, si $t x=0, x \in E$, on a $t X_{i} x=0$ pour tout $i$, d'où $X_{i} x=0$ pour tout $i$ (d'après (c)), i.e. $I x=0$, et $x=0$. On en déduit une suite exacte:

$$
0 \rightarrow E \stackrel{t}{\rightarrow} E \rightarrow E / t E \rightarrow 0,
$$

d'où une suite exacte d'homologie:

$$
H_{i}(E) \stackrel{t}{\rightarrow} H_{i}(E) \rightarrow H_{i}(E / t E) \rightarrow \cdots .
$$

Mais une propriété élémentaire des $H_{i}$ montre que $t$ annule les $H_{i}$. La grande suite exacte précédente se coupe donc en petits morceaux de la forme:

$$
0 \rightarrow H_{i}(E) \rightarrow H_{i}(E / t E) \rightarrow H_{i-1}(E) \rightarrow 0 .
$$

Commes les homomorphismes $H_{i}(E) \rightarrow H_{i}(E / t E)$ et $H_{i}(E / t E) \rightarrow \cdots$ conservent les degrés, on peut partout remplacer $H_{i}$ par $H_{i}^{+}$, d'où le lemme.

ThÉOR̀̀me. Soient $\left(t_{1}, \cdots, t_{m}\right) m$ combinaisons linéaires des coordonnées $X_{i}$. On suppose que pour tout $i, 1 \leqq i \leqq m$, le couple formé de $E_{i-1}=E /\left(t_{1}, \cdots, t_{i-1}\right) E$ et de $t_{i}$ vérifie la condition (c). On a alors: 


$$
\begin{aligned}
& H_{i}(E)^{+}=0 \text { pour } i>n-m, \\
& H_{n-m}(E)^{+}=H_{n-m+1}\left(E_{1}\right)^{+}=\cdots=H_{n}\left(E_{m}\right)^{+} .
\end{aligned}
$$

[L'hypothèse signifie que les relations $t_{i} x=0, x \in E /\left(t_{1}, \cdots, t_{i-1}\right) E$ entraînent $\operatorname{deg}(x)=0$.]

On raisonne par récurrence sur $m$, le cas $m=0$ étant évident (et le cas $m=1$ étant conséquence facile des Lemmes 1 et 2 ). L'hypothèse de récurrence montre que $H_{i}(E)^{+}=0$ pour $i>n-m+1$, et que $H_{n-m+1}(E)^{+}$ est isomorphe à $H_{n}\left(E_{m-1}\right)+$. Le Lemme 1, appliqué à $E_{m-1}$ et $t_{m}$, montre que $H_{n}\left(E_{m-1}\right)^{+}=0$. D'où $H_{n-m+1}(E)^{+}=0$. Reste à démontrer la dernière ligne, autrement dit que

$$
H_{n-m+i}\left(E_{i}\right)^{+}=H_{n-m+i+1}\left(E_{i+1}\right)^{+} .
$$

Cela résulte simplement du Lemme 2, compte tenu de ce que $H_{n-m+i+1}\left(E_{i}\right)^{+}=0$ grâce à l'hypothèse de récurrence.

Disons, pour simplifier, qu'une suite $\left(t_{1}, \cdots, t_{m}\right)$ vérifiant les conditions du théorème est une suite quasi-régulière pour $E$.

Corollaire. Pour que l'on puisse prolonger la suite quasi-régulière $\left(t_{1}, \cdots, t_{m}\right)$ en une suite quasi-régulière $\left(t_{1}, \cdots, t_{m+1}\right)$, il faut et il suffit que l'on ait $H_{n-m}(E)^{+}=0$.

Cela résulte du Lemme 1 , appliqué au module $E_{m}=E /\left(t_{1}, \cdots, t_{m}\right) E$.

On en arrive enfin aux modules "involutifs," donnés par le théorème suivant:

THÉORÈme. (1) Si E possède une suite quasi-régulière $\left(t_{1}, \cdots, t_{n}\right)$ de longueur $n$, on a $H_{i}(E)^{+}=0$ pour tout $i>0$.

(2) Inversement, si $H_{i}(E)^{+}=0$ pour tout $i \geqq 1$, toute suite $\left(t_{1}, \cdots, t_{n}\right)$ "générique" est quasi-régulière pour $E$.

C'est immédiat en appliquant le théorème et son corollaire.

REMARque. Si $E$ est engendré par ses éléments de degré zéro (ce qui est le cas chez vous), on a en plus $H_{0}(E)^{+}=0$; c'est bien plus joli (ce sont ces modules qu'on devra appeler "involutifs").

Du point de vue pratique, il serait commode de définir les involutifs par la condition homologique $H_{i}(E)^{+}=0$ pour tout $i$. Noter que, si $E$ est un module qulconque, et si l'on tronque $E$ à l'ordre $k$ en posant: $T r_{k} E=E^{k} \oplus E^{k+1} \oplus \cdots$ (avec décalage des degrés de $k$ ) le module $\operatorname{Tr}_{k} E$ est involutif pour $k$ asezg rand. Cela résulte simplement du fait que les $H_{i}(E)$ sont des espaces vectoriels de dimension finie.

Autre définition des modules involutifs: ce sont ceux qui possèdent une résolution minimale de la forme: $0 \rightarrow L_{n} \rightarrow \cdots \rightarrow L_{0} \rightarrow E \rightarrow 0$, où $L_{i}$ est un module libre gradué dont les générateurs sont homogènes 
de degré $i$. En d'autres termes, $E$ est engendré par des éléments de degré 0 , les relations entre ces générateurs sont engendrées par des éléments de degré 1 , les relations entre relations par des éléments de degré 2, etc. C'est une curiosité.

Bien à vous. J.-P. Serre

\section{BIBLIOGRAPHY}

1. U. Amaldi, Introducione alla teoria dei gruppi continui infiniti di transformazioni. I, II, Librería del Univ. de Roma, Rome, 1942, 1944.

2. D. Bernard, Sur la géométrie différentielle des G-structures, Ann. Inst. Fourier (Grenoble) 10 (1960), 151-270.

3. E. Cartan, Sur la structure des groupes infinis de transformations, Ann. Sci. Ecole Norm. Sup. 21 (1904), 153-206; 22 (1905), 219-308 or Oeuvres complètes. II, vol. 2, Groupes infinis, systèmes différentiels, theories d'equivalence, pp. 571-714, Gauthier-Villars, Paris, 1952.

4. - Les sous-groupes des groupes continus de transformations, Ann. Sci. Ecole Norm. Sup. 25 (1908), 57-194; Oeuvres completes. II, vol. 2, Groupes infinis, systèmes différentiels, thêories d'équivalence, pp. 719-856, Gauthier-Villars, Paris, 1953.

5. - Les groupes de transformations continues, infinis, simples, Ann. Sci. Ecole Norm. Sup. 26 (1909), 93-161.

6. - Oeuvres complètes. II, vol. 2, Les problèmes d'équivalence, pp. 1311-1334, Gauthier-Villars, Paris, 1953.

7. - Oeuvres complètes. II, vol. 2, La structure des groupes infinis, pp. 13351384, Gauthier-Villars, Paris, 1953.

8. - Les systèmes différentielles extérieurs et leurs applications geométriques, Actualités Sci. Ind. no. 994, Hermann, Paris, 1945.

9. S. S. Chern, Pseudo-groupes continus infinis, Géométrie différentielle. Colloques Internationaux du Centre National de la Recherche Scientifique (Strasbourg, 1953), pp. 119-136, Centre National de la Recherche Scientifique, Paris, 1953.

10. - An elementary proof of the existence of isothermal parameters on a surface, Proc. Amer. Math. Soc. 6 (1955), 771-783.

11. - On a generalization of Kähler geometry, pp. 103-121, A symposium in honor of S. Lefschetz, Princeton Univ. Press, Princeton, N. J., 1957.

12. C. Ehresmann, Structures locales et structures infinitesimales, C. R. Acad. Sci. Paris 254 (1951), 587-589.

13. - Introduction à la théorie des structures infinitésimales et des pseudogroupes de Lie, Géométrie différentielle. Colloques Internationaux du Centre National de la Recherche Scientifique (Strasbourg, 1953), pp. 97-110, Centre National de la Recherche Scientifique, Paris, 1953.

14. —- Structures locales, Ann. Mat. Pura Appl. (4) 36 (1954), 133-142.

15. V. W. Guillemin, The geometry of G-structures of finite type, thesis, Harvard Univ., Cambridge, Mass., 1962.

16. V. W. Guillemin and Shlomo Sternberg, Sur les systèmes de formes differentielles, Ann. Inst. Fourier (Grenoble) (to appear).

17. E. Kähler, Einfuhrung in die Theorie der Systeme von Differentialgleichungen, Chelsea, New York, 1949.

18. M. Kuranishi, On E. Cartan's prolongation theorem of exterior differential systems, Amer. J. Math. 79 (1957), 1-47. 
19. - On the local theory of continuous infinite pseudo-groups. I, II, Nagoya Math. J. 15 (1959), 225-260; 19 (1961), 55-91.

20. P. Libermann, Sur le problème d'équivalence de certaines structures infinitésimales, Ann. Mat. Pura Appl. (4) 36 (1954), 27-120.

21. —, Pseudogroupes infinitésimales, C. R. Acad. Sci. Paris 246 (1958), 13651368.

22. S. Lie, Gesammelte Abhandlungen, Band VI, pp. 300-365, Orlo, Leipzig, 1927.

23. Y. Matsushima, Pseudogroupes de Lie transitifs, Séminaire Bourbaki, no. 118, Hermann, Paris, 1955.

24. —— Sur les algèbres de Lie linéaires semi-involutives, Colloque de topologie de Strasbourg, 1954.

25. A. Newlander and L. Nirenberg, Complex analytic coordinates in almost complex manifolds, Ann. of Math. (2) 65 (1957), 391-404.

26. J.-P. Serre, Algèbre locale, mimeographed notes, Paris.

27. I. M. Singer and S. Sternberg, On the infinite groups of Lie and Cartan. I (to appear).

28. D. C. Spencer, Deformation of structures on manifolds defined by transitive continuous pseudogroups, Ann. of Math. (2) 76 (1962), 306-445.

29. S. Sternberg, Lectures on differential geometry, Prentice-Hall, Englewood Cliffs, N. J., 1964.

30. R. Utiyama, Invariant theoretical interpretation of interaction, Phys. Rev. 101 (1956), 1597-1607.

31. O. Zariski and P. Samuel, Commutative algebra, vol. II, Van Nostrand, Princeton, N. J., 1960.

COLUMBIA UNIVERSITY AND

HARVARD UNIVERSITY 